This item was submitted to Loughborough's Research Repository by the author.

Items in Figshare are protected by copyright, with all rights reserved, unless otherwise indicated.

\title{
Robust and discriminative zero-watermark scheme based on invariant features and similarity-based retrieval to protect large-scale DIBR 3D videos
}

PLEASE CITE THE PUBLISHED VERSION

https://doi.org/10.1016/j.ins.2020.06.066

\section{PUBLISHER}

Elsevier

VERSION

AM (Accepted Manuscript)

\section{PUBLISHER STATEMENT}

This paper was accepted for publication in the journal Information Sciences and the definitive published version is available at https://doi.org/10.1016/j.ins.2020.06.066.

LICENCE

CC BY-NC-ND 4.0

\section{REPOSITORY RECORD}

Liu, Xiyao, Yifan Wang, Ziqiang Sun, Lei Wang, Rongchang Zhao, Yuesheng Zhu, Beiji Zou, Yuqian Zhao, and Hui Fang. 2020. "Robust and Discriminative Zero-watermark Scheme Based on Invariant Features and Similarity-based Retrieval to Protect Large-scale DIBR 3D Videos". Loughborough University. https://hdl.handle.net/2134/12639074.v1. 


\title{
Robust and discriminative zero-watermark scheme based on invariant features and similarity-based retrieval to protect large-scale DIBR 3D videos
}

Xiyao Liu ${ }^{1}$, Yifan Wang ${ }^{1}$, Ziqiang Sun ${ }^{2 *}$, Lei Wang ${ }^{1}$, Rongchang Zhao ${ }^{1}$, Yuesheng $Z^{2}{ }^{3}$, Beiji Zou ${ }^{1}$, Yuqian Zhao ${ }^{4}$ and Hui Fang ${ }^{5}$

1. School of Computer Science and Engineering, Central South University, Changsha, 410083, China.

2. Nanjing Research Institute of Electronics Technology, Nanjing, 210039, China.

3. Shenzhen Graduate School, Peking University, Shenzhen, 518055, China.

4. School of Automation, Central South University, Changsha, 410083, China.

5. Computer Science Department, Loughborough University, Loughborough, LE11 3TU, UK.

E-mail:1xyzoewx@csu.edu.cn; sunziq87@163.comＴelephone:+8615111327037;+8615951832106

\begin{abstract}
Digital rights management (DRM) of depth-image-based rendering (DIBR) 3D video is an emerging area of research. Existing schemes for DIBR 3D video cause video distortions, are vulnerable to severe signal and geometric attacks, cannot protect 2D frames and depth maps independently, or have difficulty handling large-scale videos. To address these issues, a novel zero-watermark scheme based on invariant features and similarity-based retrieval to protect DIBR 3D video (RZW-SR) is proposed in this study. In RZW-SR, invariant features are extracted to generate master and ownership shares to provide distortion-free, robust and discriminative copyright identification under various attacks. Different from conventional zero-watermark schemes, our proposed scheme stores features and ownership shares correlatively and designs a similarity-based retrieval phase to provide effective solutions for large-scale videos. In addition, flexible mechanisms based on attention-based fusion are designed to protect 2D frames and depth maps, either independently or simultaneously. The experimental results demonstrate that RZW-SR has superior DRM performance compared to existing schemes. First, RZW-SR can obtain the ownership shares relevant to a particular 3D video precisely and reliably for effective copyright identification of large-scale videos. Second, RZW-SR ensures lossless, precise, reliable and flexible copyright identification for 2D frames and depth maps of 3D videos.
\end{abstract}

Keywords: DIBR 3D video; zero-watermark scheme; invariant feature; similarity-based retrieval; attention-based fusion; copyright identification 


\section{Introduction}

Protecting the copyrights of three-dimensional (3D) videos has become a crucial issue $[4,26,33,45]$. On the one hand, the risk of copyright infringement for $3 \mathrm{D}$ videos has increased because $3 \mathrm{D}$ videos, which can provide better immersive experiences to viewers than traditional 2D videos, have become more and more popular over the Internet $[22,32,40,46]$. On the other hand, the illegal copying and distribution of 3D videos will cause more serious losses to their owners than that of $2 \mathrm{D}$ videos because the production costs of 3D videos are much higher.

In this study, DIBR 3D video is chosen as the protection target for the following two reasons. First, 3D videos stored in DIBR format can reduce storage and transmission-bandwidth requirements compared with stereoscopic format $[31,40]$. Second, DIBR is a key technique for converting existing 2D videos to 3D videos, and 3D videos synthesized using DIBR have much lower costs than those using stereo videos because they do not require different cameras to capture video frames from various viewpoints.

Compared with the protection of traditional 2D videos, the protection of DIBR 3D videos is more complex. Original 2D frames with depth maps in 3D videos can converted into synthesized frames. Therefore, the watermark should be obtained from either the original 2D frames, synthesized frames or depth map. Because synthesized frames after DIBR conversion are different from original 2D frames and their pixels are shifted horizontally compared to the original, the protection scheme for 3D videos should be DIBR invariant. In addition, producers of 2D frames and depth maps are possibly different if $3 \mathrm{D}$ videos are synthesized from existing 2D videos. In this situation, copyrights of 2D frames and depth maps should be protected independently. Otherwise, they should be protected simultaneously.

Watermarking is one of the most popular methodologies for handling digital rights management (DRM) issues $[4,25,39,44]$. The existing watermark schemes for DRM of DIBR-based 3D videos, which can mainly be classified into 2D frame-based watermark, depth map-based watermark and zero-watermark schemes, still have room for improvement: 1) 2D frame-based watermark schemes [1-3, 6-7, 9, 13, 20, 23, $26,31,34,36,47]$, which only embed watermarks into 2D frames, introduce irreversible distortions to the video content [33]. In addition, they ignore the situation in which the producers of 2D frames and depth maps may be different and thus cannot protect the copyright of depth maps independently. 2) For the depth map-based watermark schemes $[8,28-29,33,37-38,42]$, which only embed watermark into depth maps, their robustness against severe signal attacks and geometrical attacks is insufficient [30]. In addition, they cannot protect the copyright of 2D frames independently. Finally, they cannot extract any copyright information from the $3 \mathrm{D}$ videos transmitted and stored by two synthesized frames. 3) Zero-watermark 
schemes $[30,45]$ generate ownership shares, which indicate the mapping relationships between video features and the watermark, without any direct watermark embedding. Zero-watermark schemes are more promising than the other two classes of watermarking schemes because they are distortion-free and robust against many types of severe attacks. However, zero-watermark schemes are based on the premise that the precise ownership share relevant to a video has already been obtained for effective copyright identification. Otherwise, the copyright of this video cannot be identified correctly, and the verification of an illegal copy would fail. This still needs improvement because it is difficult for zero-watermark to obtain the precise ownership share when processing large-scale 3D videos due to the lack of an efficient indexing mechanism. Fortunately, it is noted that the methods based on manifold similarity and retrieval $[14-16,49]$ have the potential to address this problem.

To overcome the abovementioned disadvantages, we propose a novel zero-watermark scheme for copyright protection of DIBR 3D video (RZW-SR) by fusing the similarity-based retrieval into the architecture of conventional zero-watermark in this study. In our proposed scheme, features of 2D frames and depth maps are first extracted by calculating the centroids of normalized variations between temporally informative representative images (TIRI) [11] and video frames. These TIRI-variation-centroid-based (TIRIVC) features are sufficiently robust and discriminative. Then, the master shares and ownership shares are generated based on these features and the watermark information according to the visual secret sharing (VSS) method [35]. The extracted features and their relevant ownership shares are stored, and the injective index relationships between them are established. For a queried 3D video, the similarity-based retrieval phase is first executed, in which its features are extracted and compared with the stored features, which is not performed in the conventional zero-watermark schemes. If any match occurs, the master shares are then generated from the extracted features. Finally, the copyright ownership of an illegal 3D video is identified by stacking the generated master shares with the ownership shares obtained according to the retrieval results. For both similarity-based retrieval and copyright identification, flexible mechanisms are designed. When the copyrights of 2D frames and depth maps are different, they are retrieved separately, and their copyrights are identified independently. Otherwise, the retrieval and copyright identification results are fused based on an attention-based fusion method [18] to enhance the DRM performance.

The key contributions of our proposed scheme are as follows:

1) To the best of our knowledge, it is the first scheme to fuse similarity-based retrieval with a zero-watermark technique to protect large-scale DIBR 3D videos.

2) TIRIVC features are designed to ensure the distinguishability of different $3 \mathrm{D}$ videos and robustness 
against various signal, geometrical and DIBR attacks for effective similarity-based retrieval and copyright identification.

3) Flexible mechanisms using attention-based fusion are designed for both similarity-based retrieval and copyright identification to better satisfy the requirements for protecting DIBR 3D videos.

4) Comprehensive comparisons are made to demonstrate the superiority of our proposed scheme compared with the state-of-the-art watermark schemes for protecting DIBR 3D videos.

Fig. 1 summarizes how the proposed RZW-SR differs from existing 2D frame-based watermark, depth map-based watermark and zero-watermark schemes for protecting 3D DIBR videos. On the one hand, compared with existing 2D frame-based and depth map-based watermark schemes, copyrights of both 2D frames and depth maps are identified either independently or simultaneously without any video distortion in RZW-SR. On the other hand, compared with the existing zero-watermark schemes, ownership shares relevant to a particular $3 \mathrm{D}$ video can be obtained precisely and reliably based on the similarity-based retrieval when processing large-scale videos in RZW-SR. The coloured parts of Fig. 1 emphasize these differences. Specifically, the red-coloured parts show the disadvantages of existing 2D frame-based watermark, depth map-based watermark and zero-watermark schemes, whereas the blue-coloured parts demonstrate the superiority of our proposed RZW-SR.

The rest of the paper is organized as follows. In Section II, the related works are listed. In Section III, the proposed RZW-SR is described in detail. The experimental results and analyses are presented in Section IV, and the conclusions are drawn in Section V.

\section{Related work}

Existing schemes for copyright protection of DIBR-based 3D video can be mainly classified into three categories, which are 2D frame-based watermark schemes, depth map-based watermark schemes and zero-watermark schemes. Each category is individually described below. 


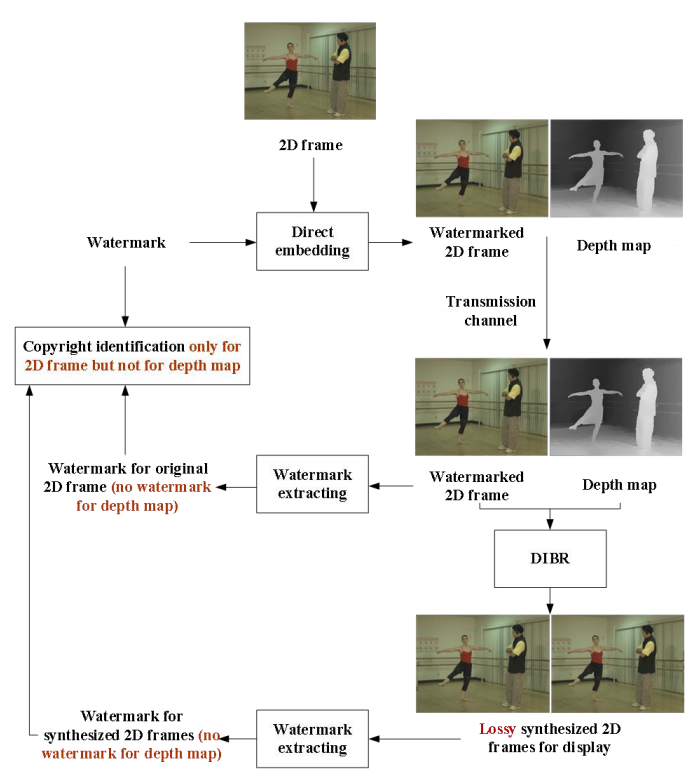

(a)

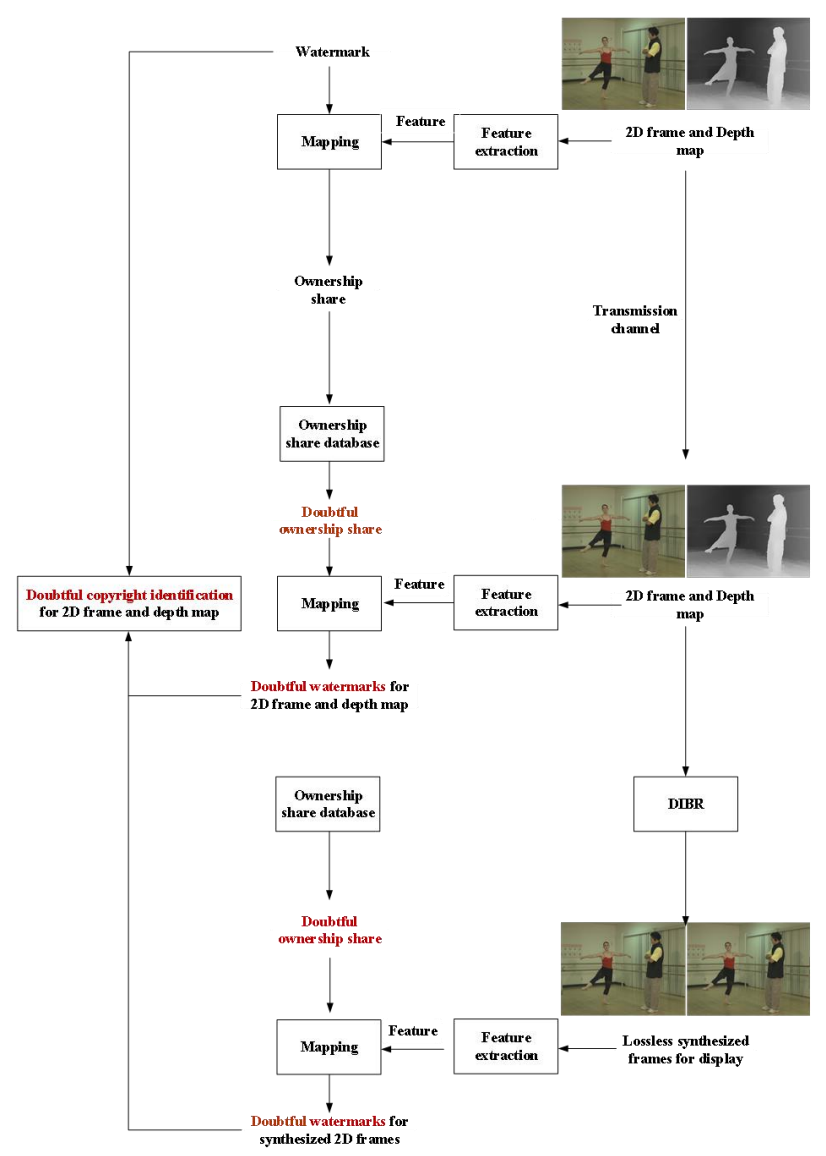

(c)

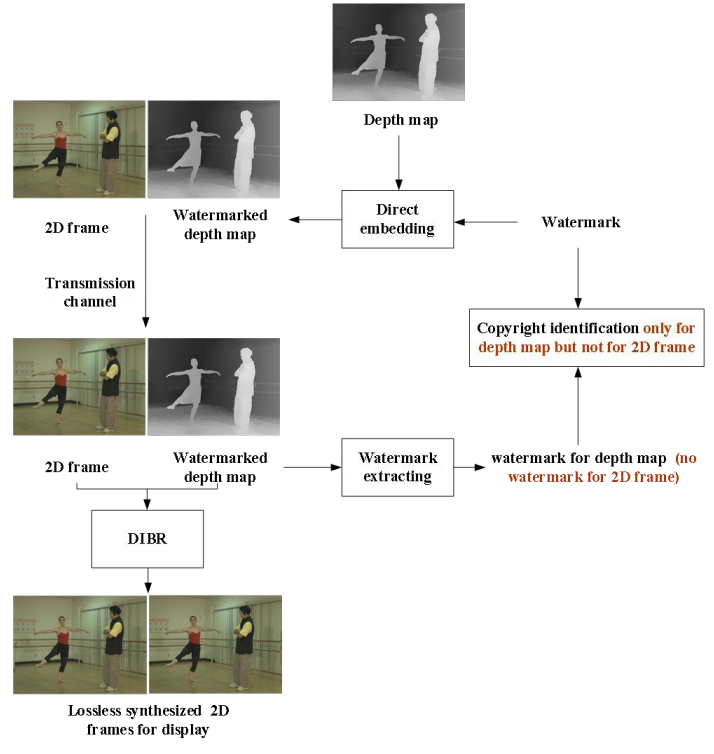

(b)

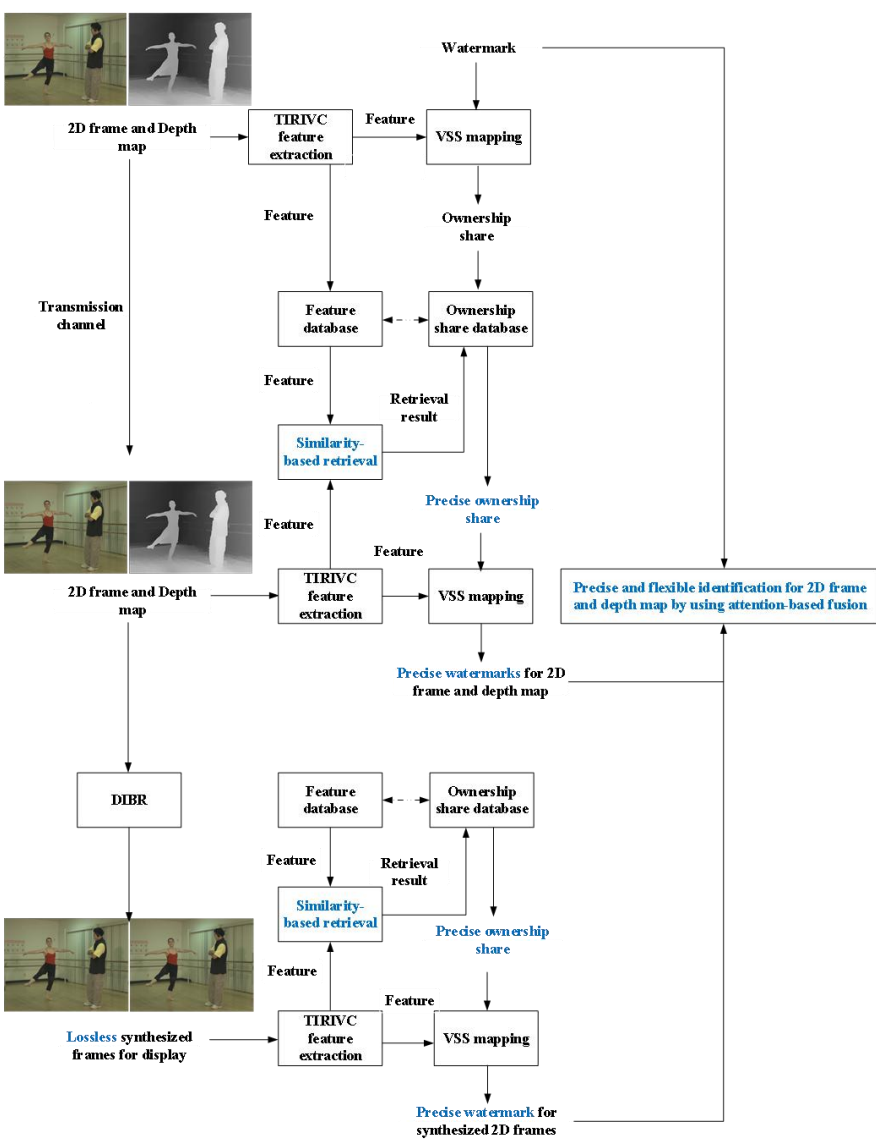

Fig. 1. Structural differences in watermark schemes: (a) 2D frame-based watermark; (b) depth map-based watermark; (c) zero-watermark; and (d) our proposed RZW-SR 


\subsection{D frame-based watermark schemes}

2D frame-based watermark schemes $[1-3,6-7,9,13,20,23,26,31,34,36,47]$ utilize the characteristics of depth maps to achieve remarkable watermark robustness and imperceptibility. Lee et al. [26] proposed a scheme utilizing the areas with high motion on the z-axis and for the pixels to be hidden by rendering for watermark embedding to ensure watermark imperceptibility. Fan et al. [13] proposed a scheme in which depth-perceptual regions of interest (DP-ROI) are constructed for watermark embedding by integrating the foreground, the depth-edge and the grey-contour regions to achieve better watermark imperceptibility. For these schemes, the watermark can hardly be extracted from the synthesized frames because the pixels are shifted horizontally by DIBR, and thus the synchronism of watermark embedding and extraction is destroyed after the DIBR process. To address this issue, DIBR invariant schemes have been designed in some works. Kim et al. [20] proposed a scheme that quantizes the coefficients of a dual-tree complex wavelet transform (DT-CWT) to make use of the approximate shift invariance and directional selectivity of DT-CWT. Asikuzzaman et al. [2-3] also proposed DT-CWT based schemes in which the watermark is embedded in the chrominance channels of 2D frames to achieve stronger robustness. Lin et al. [31] proposed a robustness watermark scheme in which multiple watermarks are embedded, and the effects of watermark embedding order were studied. Niu et al. [7, 47] proposed histogram-shape-based schemes in which suitable groups of pixel histograms are selected for watermark embedding to enhance the watermark robustness against geometrical attacks and DIBR distortions. Al-Haj et al. [1] proposed a watermark scheme based on mathematical transforms in which the discrete wavelet transform (DWT) and the singular value decomposition (SVD) are utilized to provide complementary robustness against watermarking attacks. Chen et al. [9] proposed a scheme in which the watermark is embedded into contourlet subbands of the 2D frame to make use of the directional multiresolution image representation and convenient tree structures of the contourlet transform. Lee et al. [34] proposed a scheme in which embedding locations are selected based on a depth variation prediction map and watermarks are embedded in the 2D DWT domain by quantization index modulation (QIM). To further enhance the robustness against geometric attacks, several watermark schemes based on geometric resynchronization functions $[6,23,36]$ have been proposed. In these schemes, the resynchronizations between watermarked and attacked video frames are achieved by embedding a pre-defined template [23] or utilizing local feature points $[6,36]$, such as scale-invariant feature transform (SIFT) descriptors. 


\subsection{Depth map-based watermark schemes}

Depth map-based watermark schemes $[8,28-29,33,37-38,42]$ restrict the modifications of depth maps below a certain threshold to keep the synthesized 3D videos undistorted. One category of depth map-based schemes is based on the unseen visible watermark (UVW) technique [10, 17]. Lin et al. [33] and Pei et al. [37-38] proposed several schemes under this category. In these schemes, the synthesized 3D videos are undistorted under normal viewing conditions, and meaningful watermarks can be recognized when changing the rendering parameters. However, UVW-based watermarks are only viewable for subjective copyright identification and can hardly be extracted for objective copyright identification. In addition, the modifications of depth maps are restricted based on prior estimations, which limit the selection of watermark-embedding methods and thus weaken the watermark robustness. To address these two issues, Liu et al. [29] proposed an advanced unseen extractable watermark (AUEW) scheme. In this scheme, watermarks are embedded by quantizing the sum of AC coefficients in the pseudo 3D-DCT domain and can be directly extracted for objective copyright identification. Moreover, simulations of the embedding process rather than prior estimations are utilized to restrict the modifications of depth maps for stronger watermark robustness. The other category of depth map-based schemes is depth-no-synthesis-error-model-based reversible watermark schemes (D-NOSE-RW) $[8,28,42]$. In these schemes, watermarks are embedded into depth maps based on reversible watermark techniques, such as difference expansion [21], error prediction [43] and histogram modification [24], for lossless recovery of depth maps. Moreover, locations are selected based on the D-NOSE model for better 3D viewing experiences. Although the depth map-based watermark schemes can ensure the losslessness of the synthesized 3D videos, they are vulnerable to attacks and thus cannot provide reliable copyright identification when $3 \mathrm{D}$ videos are attacked.

\subsection{Zero-watermark schemes}

Zero-watermark schemes generate mapping relationships between video features and watermarks without direct watermark embedding to avoid content distortion. Liu et al. [30] proposed a robust zero-watermark scheme for DIBR 3D video (RZW the 2D-DCT coefficients of temporally informative representative images (TIRI) [11] of 2D frames and depth maps. Then, the ownership shares are generated based on $(2,2)$ VSS and stored for copyright identification instead of embedding watermarks into the video content. The copyrights of a queried 3D video are recovered by stacking the master shares and ownership shares. By utilizing the robust features of both 2D frames and depth maps, not only can the robustness of the zero-watermark scheme against various attacks be 
ensured but also the copyrights of 2D frames and depth maps can be identified either independently or simultaneously to satisfy the different DRM requirements of DIBR 3D videos. However, the robustness of RZW $_{\text {DIBR }}$ against geometrical attacks still needs improvements. Wang et al. [45] designed another robust zero-watermark scheme for stereo 3D video $\left(\mathrm{RZW}_{\text {stereo }}\right)$. In this scheme, features are extracted from the two stereo 2D frames based on ternary number theory and radial harmonic Fourier moments (TRRHFM). Although this scheme is robust against various signal attacks and geometrical attacks, it does not offer a protection solution for depth maps when it is used for DIBR 3D videos and thus cannot fully satisfy the DRM requirements when the copyrights of the $2 \mathrm{D}$ frames and depth maps are different. In addition, the distinguishability of TRRHFM-based features is not sufficient.

Although the existing zero-watermark schemes show more promising performances compared with 2D frame-based and depth map-based watermark schemes in terms of video quality or watermarking robustness, neither of them can obtain the precise ownership share effectively when processing large-scale 3D videos because no efficient indexing mechanism is designed in these zero-watermark schemes. In addition, neither of the existing zero-watermark schemes can guarantee sufficient robustness against geometrical attacks and distinguishability for different 3D videos at the same time. Our proposed RZW-SR scheme fuses the similarity-based retrieval with zero-watermark techniques to handle large-scale 3D videos. In addition, RZW-SR designs novel robust and discriminative features with flexible retrieval and copyright identification mechanisms to ensure flexible, reliable and precise copyright identification at the same time. Our proposed RZW-SR is explained in detail in the following section.

\section{Proposed scheme}

The proposed scheme includes three phases, which are the copyright-registration phase, similarity-based retrieval phase, and copyright-identification phase, as shown in Fig. 2. Each phase is individually described below.

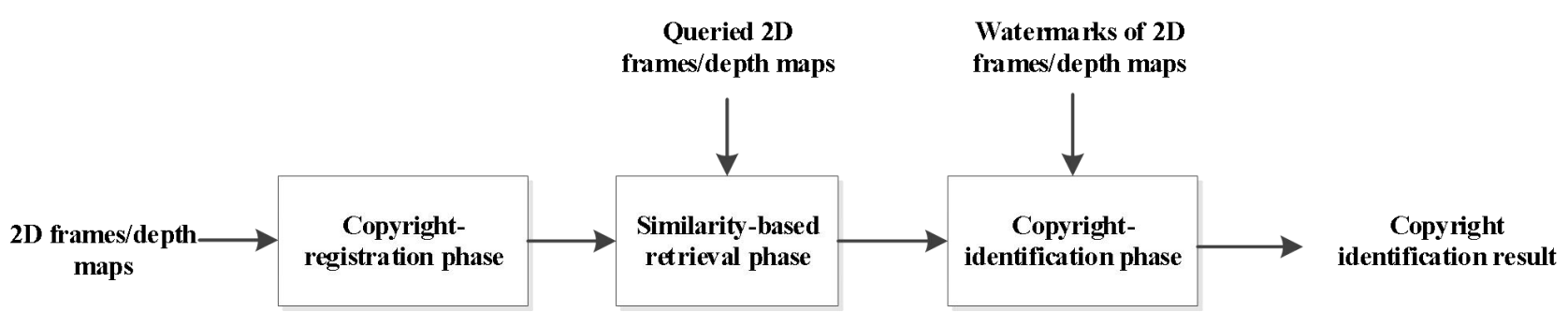

Fig. 2. Procedure of our proposed scheme 


\subsection{Copyright-registration phase}

In the copyright-registration phase, feature and ownership-share databases of both $2 \mathrm{D}$ frames and depth maps are constructed from their features and watermark information, as shown in Fig. 3.

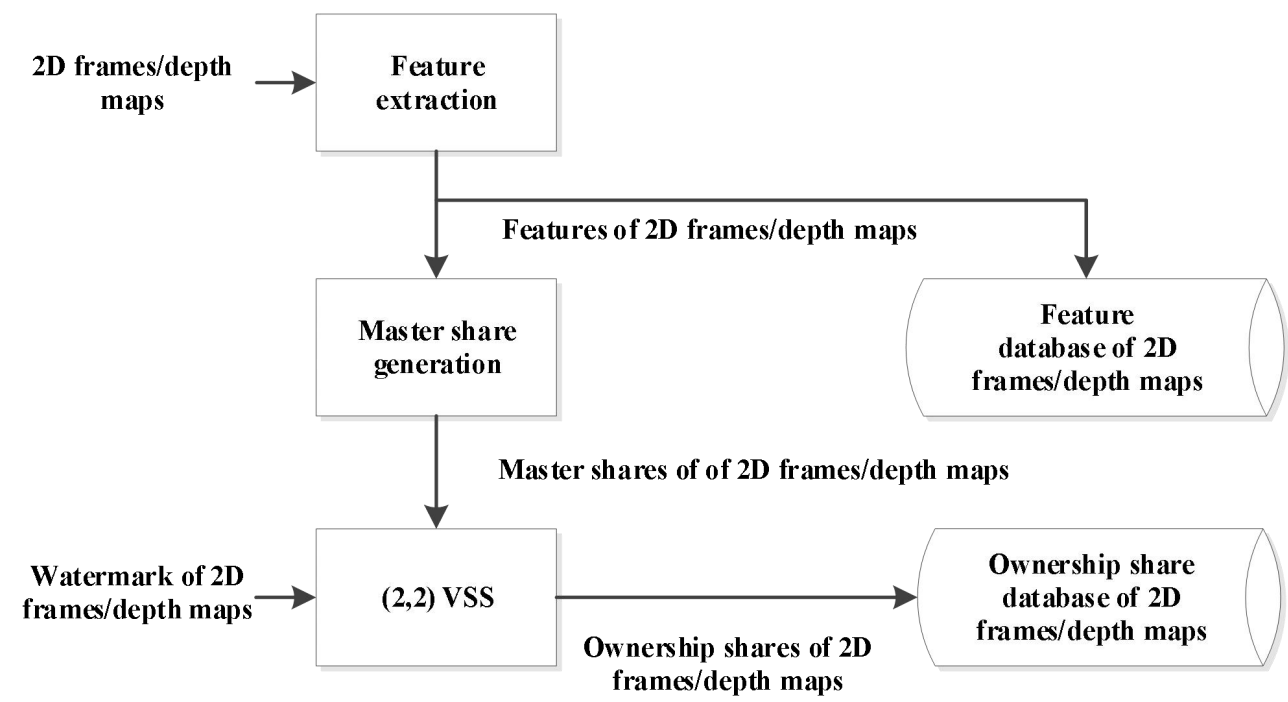

Fig. 3. Copyright-registration phase of our proposed scheme

\subsubsection{Feature extraction}

In our study, the same feature-extraction methods of 2D frames and depth maps are designed. The procedures of feature extraction are shown in Fig. 4 and listed below.

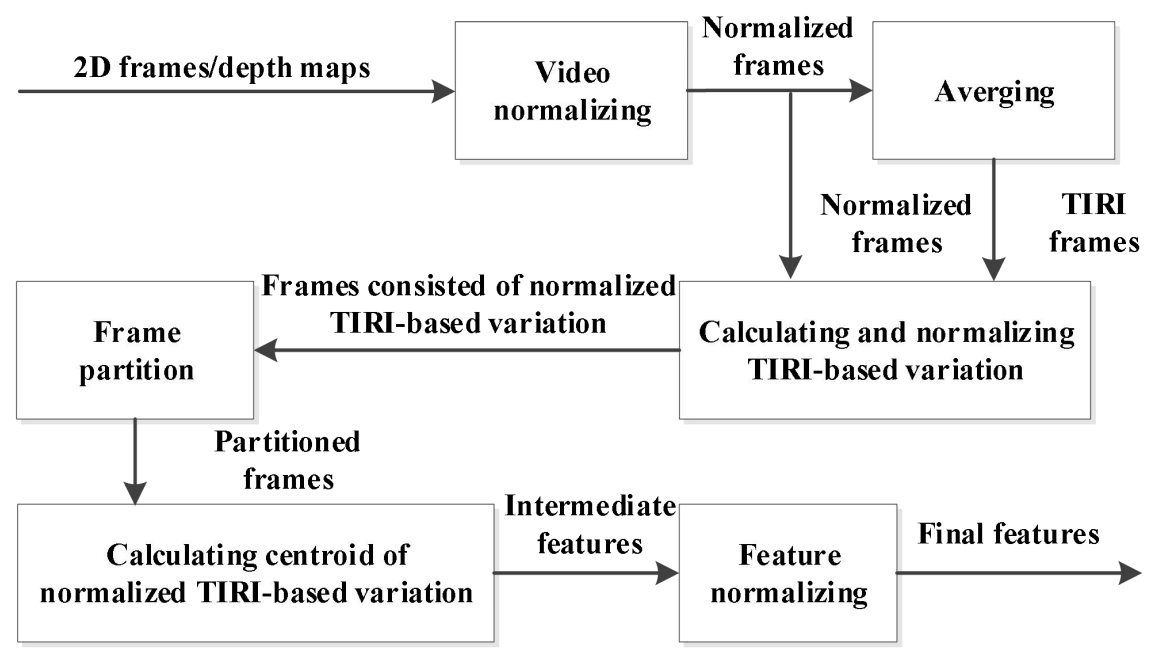

Fig. 4. Feature extraction of our proposed scheme

Step 1. Normalize both the 2 D frames and depth maps, from $w \times h \times l$ to $320 \times 320 \times 100$ based on spatial and temporal resampling and smoothing. Here, $w$ and $h$ are the width and height of the original video frames, respectively. $l$ is the frame number of the processed 3D video clip. Select the luminance of the normalized 2D frames and depth maps, denoted as $R_{2 d}$ and $R_{d e p t h}$, for feature extraction. In this manner, the robustness of extracted features against resizing and temporal attacks can be enhanced.

Step 2. Subsample and average $R_{2 d}$ and $R_{\text {depth }}$ in the temporal domain to construct their TIRIs, denoted 
as $T I R I_{2 d}$ and $T I R I_{d e p t h}$, respectively, in (1).

$$
\begin{gathered}
\operatorname{TIRI}_{c}(i, j)=\sum_{m=1}^{M} R_{c}(i, j, k) \times w_{k} / \sum_{m=1}^{M} w_{k} ; \\
w_{k}=a^{k}, k=5 \times m .
\end{gathered}
$$

where $c \in\left(2 d\right.$, depth), $\operatorname{TIRI}_{2 d}(i, j)$ and $\operatorname{TIRI}_{\text {depth }}(i, j)$ are the pixels in the $i$ th row and $j$ th column of the TIRIs of 2D frames and depth maps, respectively. $R_{2 d}(i, j, k)$ and $R_{d e p t h}(i, j, k)$ are the pixels in the $i$ th row and $j$ th column of the $k_{\text {th }}$ frame of normalized 2D frames and depth maps, $w_{k}$ is the weight associated with $R_{2 d}(i, j, k)$ and $R_{\text {depth }}(i, j, k), 1 \leq i \leq 320,1 \leq j \leq 320,1 \leq m \leq 20, M=20$, and $a$ is between 0 and 1 . When $a$ is close to 1 , the generated TIRI is more similar to an averaged image and contains more temporal information. Therefore, the feature generated from the TIRI is more robust. Otherwise, the generated TIRI contains more spatial information but less temporal information, and thus the feature generated from the TIRI is more discriminative. In our study, $a$ is selected to be 1 . In this manner, the generated TIRI contains more temporal information, and thus the feature robustness is stronger against noise addition and temporal attacks.

Step 3. Generate TIRI-based variations $D_{2 d}(i, j, k)$ and $D_{\text {depth }}(i, j, k)$ by calculating the maximal absolute differences between the central pixels in normalized frames and the 8 spatial neighbourhood pixels in the TIRIs, as shown in (2).

$$
D_{c}(i, j, k)=\max \left(\left|\operatorname{TIR} I_{c}(i \pm 1, j \pm 1)-R_{c}(i, j, k)\right|\right) ;
$$

where $c \in(2 d$, depth), $2 \leq i \leq 319,2 \leq j \leq 319$ and $1 \leq k \leq 100$.

When a video frame suffers geometrical attacks, such as rotation or flipping, the 8 spatial neighbourhoods of each pixel are still located around the central pixel. Only their relative positions are modified, which does not greatly affect the maximal absolute differences calculated in (2). Therefore, the utilization of 8 spatial-neighbourhood pixels enhances the robustness against rotation and flipping attacks.

The neighbourhood pixels in TIRIs rather than those in the normalized frames are utilized to exploit temporal properties and relationships of video frames. In this manner, the feature robustness against noise addition and temporal attacks is enhanced. In contrast, the central pixels in the normalized video frames rather than those in TIRIs are utilized to exploit more detailed spatial properties of video sequences. In this manner, the feature distinguishability is enhanced.

Step 4. Normalize the TIRI-based variations, denoted as $N_{2 d}(i, j, k)$ and $N_{\text {depth }}(i, j, k)$, using (3).

$$
N_{c}(i, j, k)=\arctan \left(D_{c}(i, j, k) / T I R I_{c}(i, j)\right) \text {; }
$$

where $c \in(2 d$, depth $)$. In this manner, the feature is more robust when against global attacks such as contrast modification, brightness modification and gamma transform.

Step 5. Partition the frames consisting of normalized TIRI-based variations into a central circle and $N-1$ 
concentric rings. The radius of the central circle and the widths of concentric rings are set to $r$.

For each pixel $(i, j, k)$ in the $k_{\text {th }}$ frame, its distance $\operatorname{Dist}(i, j, k)$ to the centre point of this frame $\left(i_{o}, j_{o}, k\right)$ is first calculated as shown in (4).

$$
\operatorname{Dist}(i, j, k)=\sqrt{\left(i-i_{o}\right)^{2}+\left(j-j_{o}\right)^{2}}
$$

Then, partition $n$ of pixel $(x, y, k)$ is calculated based on $\operatorname{Dist}(i, j, k)$, as shown in (5).

$$
n=\lfloor\operatorname{Dist}(i, j, k) / r\rfloor
$$

In our study, the size of frames consisting of normalized TIRI-based variations is $320^{\times} 320, N$ equals 16 , and $r$ equals 10 . When the videos are rotated or flipped, the pixels partitioned in this manner still belong to their original associated circle or ring partitions. Therefore, feature robustness against rotation and flipping attacks is ensured. In addition, the regions outside the largest ring are not utilized in our study due to the following two reasons. On the one hand, the main visual of a video frame is usually concentrated in its central region. Therefore, features generated by discarding the regions outside the largest ring do not lose much important visual information. On the other hand, because these regions are the most common places for logo insertion and edge cropping attacks, the feature robustness against these attacks can be enhanced by discarding the regions outside the largest ring.

Step 6. Take the pixel values in TIRIs as the weights of normalized TIRI-based variations. Calculate the centroids of normalized TIRI-based variations in each partition using (6) and generate the intermediate features of 2D frames and depth maps, denoted as $f_{2 d}$ and $f_{\text {depth }}$, using (7).

$$
\begin{gathered}
\sum_{c}(n, k)=\frac{\sum_{(n-1) r \leq D i s t(i, j, k) \leq n r} \operatorname{TIRI}_{c}(i, j) N_{c}(i, j, k)}{\sum_{(n-1) r \leq D i s t(i, j, k) \leq n r} \operatorname{TIRI}_{c}(i, j)} ; \\
f_{c}=\left[v_{c}(1,1) \ldots v_{c}(N, 1) \ldots v_{c}(1, K) \ldots v_{c}(N, K)\right] ;
\end{gathered}
$$

where $c \in(2 d$, depth $)$ and $K$ is the number of frames consisting of normalized TIRI-based variations. In our study, $K$ equals 100 , and the dimensionality of $f_{2 d}$ and $f_{\text {depth }}$ is $K \times N$ and equals 1600 .

Step 7. Normalize the intermediate features by their mean and standard deviation as shown in (8) to generate the final features, $f n_{2 d}$ and $f n_{\text {depth }}$. Store $f n_{2 d}$ and $f n_{\text {depth }}$ in the 2D frame and depth map feature databases, respectively, for similarity-based retrieval and copyright identification. Here, $c \in(2 d$, depth). 


$$
\begin{aligned}
& \mu_{c}=\frac{1}{N K} \sum_{i=1}^{N K} f_{c}(i) ; \\
& \sigma_{c}=\sqrt{\frac{1}{N K-1} \sum_{i=1}^{N K}\left(f_{c}(i)-\mu_{c}\right)^{2}} ; \\
& f n_{c}(i)=\frac{f_{c}(i)-\mu_{c}}{\sigma_{c}} ;
\end{aligned}
$$

\subsubsection{Generation of master shares and ownership shares}

$(m, n)$ VSS (where $m \leq n)$ was proposed by Naor et al. [35]. In VSS, one binary image is split into $n$ shares and can be recovered from $l$ shares when $l \geq m$. Otherwise, the image cannot be recovered. $(2,2)$ VSS is a typical VSS, in which each pixel of a binary image is substituted by a pair of shares consisting of four sub-pixels. A white pixel is split into two identical shares, whereas a black pixel is split into two complementary shares.

(2-2) VSS is a low-cost injective function and utilized in our RZW-SR to generate the master shares and ownership shares. In this manner, mapping relationships between features and copyright information is injective. Therefore, robustness and distinguishability of features can be maintained during the procedures of generating master shares and ownership shares. The detailed procedures of generating master shares and ownership shares are listed below.

Step 1. Binarize the features of $2 \mathrm{D}$ frames and depth maps by their median values to construct intermediate vectors, as shown in (9).

$$
\begin{aligned}
& I_{c}(i)=1, \text { if } f n_{c}(i)>t_{c} ; \\
& I_{c}(i)=0, \text { if } f n_{c}(i) \leq t_{c}
\end{aligned}
$$

where $c \in(2 d$, depth $), 1 \leq i \leq K \times N, t_{2 d}$ is the median value of $f_{2 d}$, and $t_{\text {depth }}$ is the median value of $f_{\text {depth }}$.

Step 2. Rearrange the intermediate vectors $I_{2 d}$ and $I_{d e p t h}$ to construct intermediate matrices $V_{2 d}$ and $V_{\text {depth }}$, which are of size $40 \times 40$. Generate the master shares of 2D frames and depth maps, which are denoted as $M_{2 d}$ and $M_{\text {depth }}$, according to the $(2,2)$ VSS as shown in (10).

$$
m_{c}(i, j)=\left\{\begin{array}{l}
{\left[\begin{array}{l}
1,0 \\
0,1
\end{array}\right], V_{c}(i, j)=1} \\
{\left[\begin{array}{l}
0,1 \\
1,0
\end{array}\right], V_{c}(i, j)=0}
\end{array}\right.
$$

where $c \in(2 d$, depth $) ; m_{2 d}(i, j)$ and $m_{\text {depth }}(i, j)$ are non-overlapping $2 \times 2$ blocks of $M_{2 d}$ and $M_{d e p t h} ; 1 \leq i \leq 40$ and $1 \leq j \leq 40$.

Step 3. Generate ownership shares of 2D frames and depth maps, which are denoted as $O_{2 d}$ and $O_{\text {depth }}$, 
according to the $(2,2)$ VSS as shown in (11).

$$
o_{c}(i, j)=\left\{\begin{array}{l}
{\left[\begin{array}{l}
1,0 \\
0,1
\end{array}\right], \text { if } m_{c}(i, j)=\left[\begin{array}{l}
1,0 \\
0,1
\end{array}\right], W_{c}(i, j)=1 ;} \\
{\left[\begin{array}{l}
0,1 \\
1,0
\end{array}\right], \text { if } m_{c}(i, j)=\left[\begin{array}{l}
1,0 \\
0,1
\end{array}\right], W_{c}(i, j)=0 ;} \\
{\left[\begin{array}{l}
0,1 \\
1,0
\end{array}\right], \text { if } m_{c}(i, j)=\left[\begin{array}{l}
0,1 \\
1,0
\end{array}\right], W_{c}(i, j)=1 ;} \\
{\left[\begin{array}{l}
1,0 \\
0,1
\end{array}\right], \text { if } m_{c}(i, j)=\left[\begin{array}{l}
0,1 \\
1,0
\end{array}\right], W_{c}(i, j)=0 ;}
\end{array}\right.
$$

where $c \in(2 d$, depth $) ; o_{2 d}(i, j)$ and $o_{\text {depth }}(i, j)$ are non-overlapping $2 \times 2$ blocks of $O_{2 d}$ and $O_{d e p t h} ; W_{2 d}(i, j)$ and $W_{\text {depth }}(i, j)$ are the watermark bits of the $2 \mathrm{D}$ frame and depth map; $1 \leq i \leq 40$ and $1 \leq j \leq 40$.

Step 4. Store the generated $O_{2 d}$ and $O_{\text {depth }}$ in ownership databases and establish injective index relationships between these ownership shares and their relevant features, which have already been stored in feature databases, for copyright identification.

\subsection{Similarity-based retrieval phase}

When a $3 \mathrm{D}$ video is queried, a similarity-based retrieval phase is executed to find the stored features that match the features of the queried video, as shown in Fig. 5.

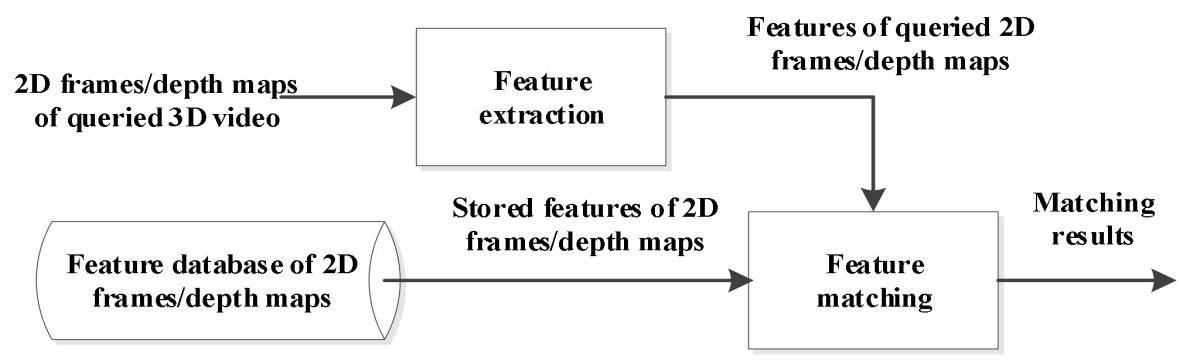

Fig. 5. Similarity-based retrieval phase of our proposed scheme

Features of queried 2D frames and depth maps, which are denoted as $f n^{\prime}{ }_{2 d}$ and $f n{ }^{\prime}{ }_{\text {depth }}$, are first extracted following the same procedures in the copyright-registration phase. The normalized distances between queried features and original features in databases are measured using (12).

$$
d_{c}=\frac{1}{L} \sum_{i=1}^{L}\left(f n_{c}(i)-f n_{c}^{\prime}(i)\right)^{2}
$$

where $c \in(2 d$, depth $)$ and $L$ is the length of the features. In our study, $L$ equals $N \times K . N$ is the number of partitions and equals 16 , and $K$ is the number of frames and equals 100 .

To satisfy different DRM requirements of DIBR 3D videos, a flexible matching mechanism is designed in our study. In the case that the copyrights of 2D frames and depth maps are different, the features of 2D 
frames and depth maps are retrieved separately and independently. Given the thresholds $T_{2 d}$ and $T_{\text {depth }}$, if any $d_{2 d}$ or $d_{d e p t h}$ is smaller than $T_{2 d}$ or $T_{d e p t h}$, respectively, the 2D frames or depth maps of two DIBR 3D videos are considered to be near-duplicates and the queried DIBR 3D video is considered to be an illegal copy. Otherwise, normalized feature distances of 2D frames and depth maps are fused. It will be better if the function $F_{D}$ satisfies the heterogeneity and monotonicity for distance fusion [18]. The heterogeneity is defined in (13), and the monotonicity is defined in (14).

$$
\begin{gathered}
F_{D}\left(d_{1}, d_{2}\right)>F_{D}\left(d_{1}-\varepsilon, d_{2}+\varepsilon\right) \\
F_{D}\left(d_{1}, d_{2}\right)<F_{D}\left(d_{1}+\varepsilon, d_{2}\right) ; \\
F_{D}\left(d_{1}, d_{2}\right)<F_{D}\left(d_{1}, d_{2}+\varepsilon\right) .
\end{gathered}
$$

where $0<\varepsilon \leq d_{1} \leq d_{2} ; d_{1}$ and $d_{2}$ are distance values to be fused.

To strictly satisfy the heterogeneity and monotonicity simultaneously, an attention-based fusion function for distances is designed inspired by the method of [18] as below.

$$
\begin{gathered}
x_{1}=\frac{1}{d_{2 d}}+\frac{1}{d_{\text {depth }}} ; \\
x_{2}=\left|\frac{1}{d_{2 d}}-\frac{1}{d_{d e p t h}}\right| \\
F_{D}\left(d_{2 d}, d_{d e p t h}\right)=\frac{1}{\frac{1}{2}\left(x_{1}+\frac{1}{1+\gamma} x_{2}\right)} .
\end{gathered}
$$

where $\gamma$ is a constant and is empirically set to 0.1 in our study.

Given a threshold $T_{\text {fusion }}$, the two DIBR 3D videos are considered to be near-duplicates and the queried DIBR 3D video is considered to be an illegal copy if any distance value fused in (15) is smaller than $T_{\text {fusion }}$.

Because 2D frames have more texture information than depth maps, whereas the distribution of pixel values in the depth maps is regional, the feature robustness characteristics of $2 \mathrm{D}$ frames and depth maps are complementary to a certain extent. Therefore, the distance fused in (15) further enhances the reliability of similarity-based retrieval against different attacks.

If the queried DIBR 3D video is considered to be an illegal copy, a copyright-identification phase is implemented. Otherwise, the procedures of RZW-SR are finished.

\subsection{Copyright-identification phase}

In this phase, ownership shares, which are relevant to the matched features, are stacked with the master shares of illegal DIBR 3D videos to recover the copyright information. The detailed procedures are shown in Fig. 6 and described below. 


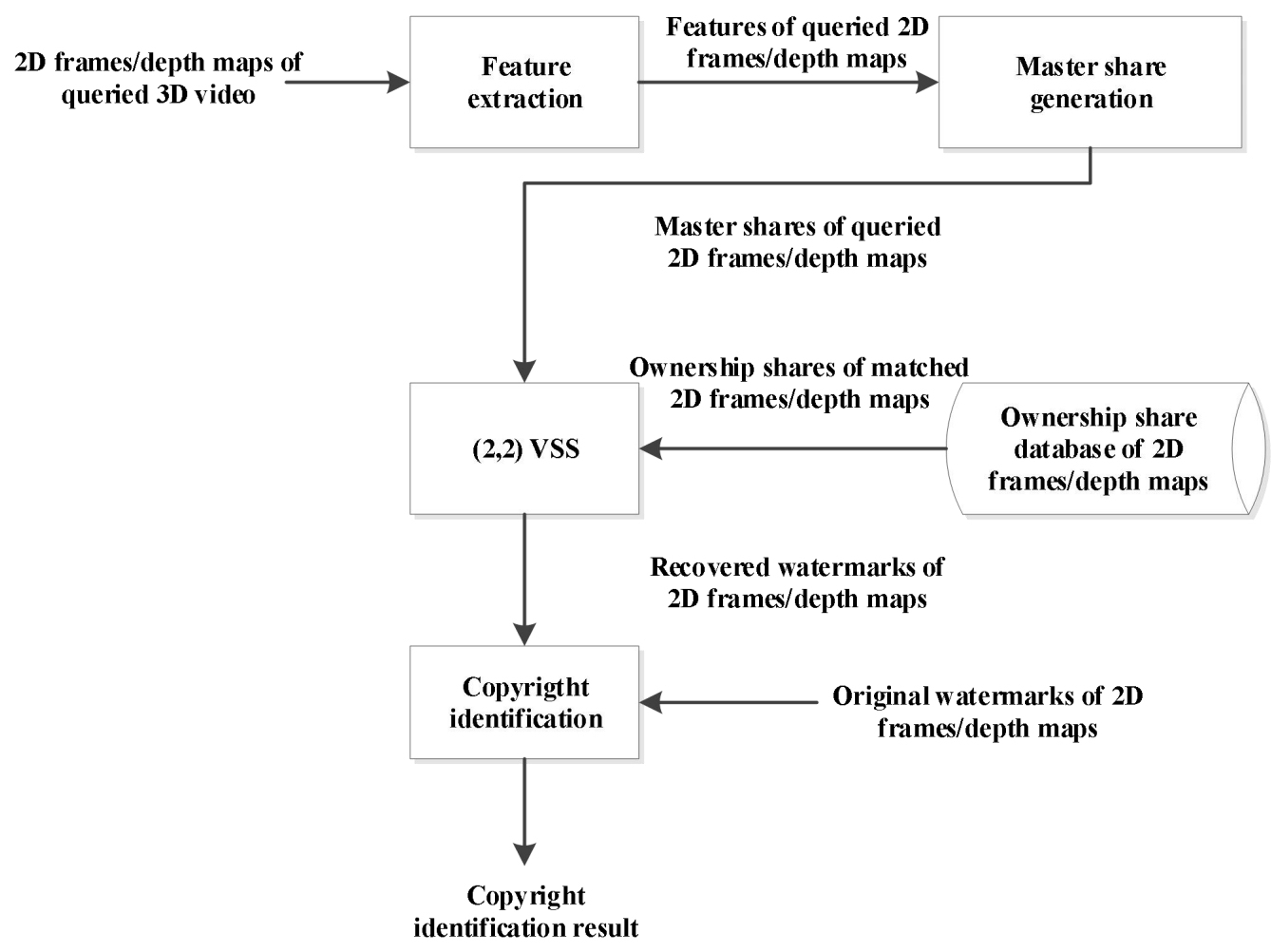

Fig. 6. Copyright-identification phase of our proposed scheme

\subsubsection{Recovery of copyright information}

Step 1. Obtain the ownership shares relevant to the matched features from ownership databases.

Step 2. Generate the master shares of illegal DIBR 3D videos, $M_{2 d}^{\prime}$ and $M^{\prime}{ }_{\text {depth }}$, from $f^{\prime}{ }_{2 d}$ and $f^{\prime}{ }_{\text {depth }}$, respectively, following the same procedure as in the copyright-registration phase.

Step 3. Stack the generated master shares with the obtained ownership shares to construct intermediate copyright-identification matrices $S_{2 d}$ and $S_{\text {depth }}$, as shown in (16).

$$
s_{c}(i, j)=\left\{\begin{array}{l}
{\left[\begin{array}{l}
1,0 \\
0,1
\end{array}\right], \text { if } m_{c}(i, j)=\left[\begin{array}{l}
1,0 \\
0,1
\end{array}\right], o_{c}(i, j)=\left[\begin{array}{l}
1,0 \\
0,1
\end{array}\right] ;} \\
{\left[\begin{array}{l}
0,0 \\
0,0
\end{array}\right], \text { if } m_{c}(i, j)=\left[\begin{array}{l}
1,0 \\
0,1
\end{array}\right], o_{c}(i, j)=\left[\begin{array}{l}
0,1 \\
1,0
\end{array}\right]} \\
{\left[\begin{array}{l}
0,0 \\
0,0
\end{array}\right], \text { if } m_{c}(i, j)=\left[\begin{array}{l}
0,1 \\
1,0
\end{array}\right], o_{c}(i, j)=\left[\begin{array}{l}
1,0 \\
0,1
\end{array}\right] ;} \\
{\left[\begin{array}{l}
0,1 \\
1,0
\end{array}\right], \text { if } m_{c}(i, j)=\left[\begin{array}{l}
0,1 \\
1,0
\end{array}\right], o_{c}(i, j)=\left[\begin{array}{l}
0,1 \\
1,0
\end{array}\right] ;}
\end{array}\right.
$$

where $c \in(2 d$,depth $) ; s_{2 d}(i, j)$ and $s_{d e p t h}(i, j)$ are non-overlapping $2 \times 2$ blocks of $S_{2 d}$ and $S_{\text {depth }} ; 1 \leq i \leq 40$ and $1 \leq j \leq 40$.

Step 4. Recover the watermark information of the $2 \mathrm{D}$ frame and depth map $W_{2 d}^{\prime}$ and $W_{d e p t h}^{\prime}$, from $S_{2 d}$ and $S_{\text {depth }}$, respectively, using (17). 


$$
W_{c}^{\prime}(i, j)= \begin{cases}1, & \sum s_{c}(i, j) \geq 2 ; \\ 0, & \sum s_{c}(i, j)<2 ;\end{cases}
$$

where $c \in(2 d$,depth $) ; 1 \leq i \leq 40,1 \leq j \leq 40,1 \leq x \leq 2$, and $1 \leq y \leq 2$.

\subsubsection{Identification of copyright ownership}

To satisfy the different DRM requirements of DIBR 3D videos, a flexible copyright-identification mechanism is designed in our study.

In the case that the copyrights of the $2 \mathrm{D}$ frames and depth maps are different, they are identified separately and independently by calculating their own bit error rates (BERs) between the original watermark and the recovered watermark as shown in (18).

$$
B E R_{c}=\frac{\sum W_{c}(i, j) \oplus W_{c}^{\prime}(i, j)}{i \times j} ;
$$

where $c \in(2 d$, depth $), 1 \leq i \leq 40$ and $1 \leq j \leq 40$.

Otherwise, the $B E R$ s of $2 \mathrm{D}$ frames and depth maps are fused. It will be better if the function $F_{B E R}$ satisfies the heterogeneity and monotonicity for BER fusion, which is similar to the distance fusion. Therefore, a similar attention-based fusion function for $B E R \mathrm{~s}$ is also designed as below:

$$
\begin{gathered}
x_{1}=\frac{1}{B E R_{2 d}}+\frac{1}{B E R_{d e p h h}} ; \\
x_{2}=\left|\frac{1}{B E R_{2 d}}-\frac{1}{B E R_{\text {depth }}}\right| \\
F_{B E R}\left(B E R_{2 d}, B E R_{\text {depth }}\right)=\frac{1}{\frac{1}{2}\left(x_{1}+\frac{1}{1+\gamma} x_{2}\right)}
\end{gathered}
$$

where $\gamma$ is a constant empirically set to 0.1 in our study.

The attention-based fusion further enhances the reliability of copyright identification because the complementary feature robustness of 2D frames and depth maps are analysed in the similarity-based retrieval phase.

\section{Experimental Result and Discussion}

\subsection{Experimental Setup}

In this study, the distinguishability and robustness of our designed feature are first evaluated in section 4.2. Then, the performances of similarity-based retrieval in our proposed RZW-SR are evaluated in section 4.3. In addition, performances of copyright identification in our proposed RZW-SR are assessed in section 4.4. 
After that, the computational complexity of our proposed RZW-SR is tested and discussed in section 4.5. Finally, qualitative comparisons between our proposed RZW-SR and other schemes for DIBR 3D videos are presented in section 4.6.

Our testing database contains 200 different DIBR 3D video clips. Some of them are from the open datasets provided by the MEPG 3DAV group [12] and Interactive Visual Media Group of Microsoft Research [50]. Others consist of 2D frames selected from existing movies, with their depth maps manually calibrated by authors based on the method in [41]. The videos in [12] consist of 125 or 250 frames at 25 fps, and the videos in [50] consist of 100 frames at 15 fps. Their frame sizes are $720 \times 576$ and $1024 \times 768$, respectively. For our manually generated DIBR video clips, they consist of 200 frames at 25 fps, and their frame sizes are $1920 \times 1080$. A watermark image of size $40 \times 40$ is utilized for copyright identification. Four sequences of synthesized frames are generated by setting the baseline distances as $5 \%$ and $7 \%$ of the video frame width. These baseline distances have been chosen carefully because baseline distances larger than 7\% may induce discomfort for 3D viewing $[1,40]$. All original 2D frames, depth maps and synthesized frames suffer 14 types of attacks, with a total of 26 different attacks. As a result, a total of 32,000 sequences of queried videos, including 5,200 sequences of attacked 2D frames, 5,200 sequences of attacked depth maps, 800 sequences of original synthesized frames and 20,800 sequences of attacked synthesized frames, are generated to evaluate performances of the proposed RZW-SR. The types and parameters of the attacks are shown in Table 1 . The $1^{\text {st }}$ to $8^{\text {th }}$ are signal attacks. The $9^{\text {th }}$ to $12^{\text {th }}$ are geometrical attacks. The last two are temporal attacks.

TABLE 1. Types of attacks

\begin{tabular}{|c|c|c|}
\hline & Types & Parameters \\
\hline \multirow{8}{*}{ Signal attacks } & Gaussian blurring (GB) & window $=9 \times 9$, variance $=1$; window $=15 \times 15$, variance $=1$ \\
\hline & Average filtering (AF) & window $=9 \times 9 ; 15 \times 15$ \\
\hline & Median filtering (MF) & window $=9 \times 9 ; 15 \times 15$ \\
\hline & Changes in contrast $(\mathrm{CC})$ & $-30 \% ;+30 \%$ \\
\hline & Changes in brightness (CB) & $-30 \% ;+30 \%$ \\
\hline & Gamma transform (GT) & $\gamma=0.6 ; 1.4$ \\
\hline & Gaussian noise addition (GN) & Mean $=0$, variance $=0.005 ;$ Mean $=0$, variance $=0.01$ \\
\hline & Logo insertion in the upper-left (LI) & Logo size $=32 \times 32 ; 64 \times 64$ \\
\hline \multirow{4}{*}{ Geometrical attacks } & Resizing (RS) & $1 / 2$ of original size; $1 / 5$ of original size \\
\hline & Crop from image edges (CR) & $5 \% ; 10 \%$ \\
\hline & Rotation (RT) & $45^{\circ} ; 90^{\circ}$ \\
\hline & Flip (FL) & Vertical, horizontal \\
\hline Temporal attacks & Frame replacing randomly (FR) & Replacing rate $=5 \%$ \\
\hline
\end{tabular}




\subsection{Evaluation of feature distinguishability and robustness}

In this section, we calculate the inter- and intra-feature distances to evaluate the feature distinguishability and robustness of the proposed RZW-SR, which indicate the accuracy and reliability of both similarity-based retrieval and copyright identification. The inter-feature distance means the feature distance between different video-clip pairs, whereas the intra-feature distance means the feature distance between the original and its attacked video. The mean inter- and intra-feature distances of our proposed RZW-SR are tested and compared with those of the other two zero-watermark schemes, which are $\mathrm{RZW}_{\text {stereo }}$ [45] and RZW $\mathrm{WIBR}_{\text {[30]. }}$ All of these distances are calculated in the same manner as shown in (12). Assuming that the features are totally independent, the theoretical value of the mean inter-feature distances of our proposed RZW-SR is 2 , which has been proved in [19]. The theoretical values of the mean inter-feature distances of RZW $\mathrm{W}_{\text {stereo }}$ [45] and RZW discriminative if the mean inter-feature distance is closer to its theoretical value. On the other hand, a feature is more robust if the mean intra-feature distance is closer to 0 . The results are listed in Table 2 .

TABLE 2. Mean inter- and intra-feature distances of different schemes

\begin{tabular}{llcc}
\hline \hline & & Mean inter-feature distances/theoretical values & Mean intra-feature distances \\
\hline $\mathrm{RZW}_{\text {stereo }}$ [45] & 2D frame & $0.1436 / 0.5000$ & 0.0311 \\
\hline \multirow{2}{*}{$\mathrm{RZW}_{\text {DIBR [30] }}$} & 2D frame & $0.4979 / 0.5000$ & 0.1456 \\
& Depth map & $0.4981 / 0.5000$ & 0.2027 \\
& Fuse together & $0.4975 / 0.5000$ & 0.1357 \\
\hline \multirow{2}{*}{ Our proposed RZW-SR } & 2D frame & $1.9080 / 2.0000$ & 0.0713 \\
& Depth map & $1.8519 / 2.0000$ & 0.0917 \\
& Fuse together & $1.6990 / 2.0000$ & 0.0337 \\
\hline \hline
\end{tabular}

As shown in Table 2, the mean inter-feature distances of our proposed RZW-SR and RZW DIBR are close to their theoretical values, whereas the mean inter-feature distance of $\mathrm{RZW}_{\text {stereo }}$ is only 0.1436 and far from its theoretical value. Furthermore, the mean intra-feature distances of our proposed RZW-SR and RZW stereo $_{\text {. }}$ are close to 0 and are much smaller than those of RZWDIBR. These results show that our designed features ensure sufficient distinguishability and robustness simultaneously and are superior to the features of $\mathrm{RZW}_{\text {stereo }}$ (not sufficiently discriminative) and RZW 


\subsection{Evaluation of similarity-based retrieval performance}

In this section, the false-negative rates $P_{f n}$ with a fixed false-positive rate $P_{f p}$ under all of these attacks are calculated to evaluate the retrieval performance of our proposed scheme. $P_{f p}$ is the probability of classifying different videos as the same and indicates the retrieval accuracy, whereas $P_{f_{n}}$ is the probability of classifying the original and its attack videos as different and indicates the retrieval reliability. $P_{f p}$ and $P_{f n}$ are defined by (20)-(21). In our experiments, $P_{f p}$ is fixed to a small value of 0.01. A retrieval function is more effective if $P_{f n}$ is closer to 0 . The results are listed in Tables 3 and 4.

TABLE 3. $P_{f n}$ with $P_{f p}=0.01$ under different attacks when utilizing original $2 \mathrm{D}$ frames, depth maps and their fusion

\begin{tabular}{|c|c|c|c|}
\hline & \multicolumn{3}{|c|}{ Our proposed RZW-SR } \\
\hline & 2D frame & Depth map & Two together \\
\hline GB $9 \times 9$ & 0.0000 & 0.0200 & 0.0000 \\
\hline GB $15 \times 15$ & 0.0000 & 0.0200 & 0.0000 \\
\hline $\mathrm{AF} 9 \times 9$ & 0.0100 & 0.0350 & 0.0000 \\
\hline AF $15 \times 15$ & 0.0100 & 0.0450 & 0.0000 \\
\hline MF $9 \times 9$ & 0.0100 & 0.0000 & 0.0000 \\
\hline MF $15 \times 15$ & 0.0100 & 0.0000 & 0.0000 \\
\hline $\mathrm{CC}-30$ & 0.0000 & 0.0000 & 0.0000 \\
\hline $\mathrm{CC}+30$ & 0.0000 & 0.0150 & 0.0000 \\
\hline $\mathrm{CB}-30$ & 0.0100 & 0.0000 & 0.0000 \\
\hline $\mathrm{CB}+30$ & 0.0000 & 0.0400 & 0.0000 \\
\hline GT $\gamma=0.6$ & 0.0000 & 0.0000 & 0.0000 \\
\hline GT $\gamma=1.4$ & 0.0000 & 0.0000 & 0.0000 \\
\hline GN 0.005 & 0.0050 & 0.0650 & 0.0000 \\
\hline GN 0.01 & 0.0050 & 0.0900 & 0.0050 \\
\hline LI $32 \times 32$ & 0.0000 & 0.0000 & 0.0000 \\
\hline LI $64 \times 64$ & 0.0000 & 0.0400 & 0.0000 \\
\hline $\mathrm{RS} 1 / 2$ & 0.0000 & 0.0000 & 0.0000 \\
\hline RS $1 / 5$ & 0.0100 & 0.0050 & 0.0000 \\
\hline CR 5\% & 0.0000 & 0.1300 & 0.0100 \\
\hline CR $10 \%$ & 0.0250 & 0.1450 & 0.0150 \\
\hline $\mathrm{RT} 45^{\circ}$ & 0.0050 & 0.1050 & 0.0000 \\
\hline RT $90^{\circ}$ & 0.0350 & 0.1450 & 0.0250 \\
\hline FL Vertical & 0.0000 & 0.0000 & 0.0000 \\
\hline FL Horizontal & 0.0000 & 0.0000 & 0.0000 \\
\hline FR $5 \%$ & 0.0100 & 0.1450 & 0.0050 \\
\hline FD 5\% & 0.0350 & 0.0250 & 0.0150 \\
\hline Average value & 0.0067 & 0.0412 & 0.0029 \\
\hline
\end{tabular}




$$
\begin{gathered}
P_{f p}=\frac{N_{f p}}{N_{d i s}} \\
P_{f n}=\frac{N_{f n}}{N_{s}}
\end{gathered}
$$

where $N_{f p}$ is the number of inter-feature distances smaller than a threshold, $N_{d i s}$ is the true number of different video clips, $N_{f n}$ is the number of intra-feature distances larger than the threshold, and $N_{s}$ is the true number of attacked videos.

Table 3 shows that all of the $P_{f n}$ of our proposed RZW-SR when utilizing original 2D frames, utilizing depth maps and fusing them together are very small, with their maximal values as $0.0350,0.1450$, and 0.0250 and average values as $0.0067,0.0412$, and 0.0029 . These results demonstrate that the similarity-based retrieval of our proposed RZW-SR is effective. In addition, $P_{f n}$ obtained when fusing the two components together are even smaller than those obtained when only utilizing original 2D frames or depth maps, which demonstrates that the attention-based fusion enhances the performances of similarity-based retrieval when the copyrights of the original 2D frames and depth maps are the same. The reason for this phenomenon is that the feature robustness characteristics of 2D frames and depth maps are complementary because 2D frames have more texture information whereas depth maps are much smoother.

Table 4 shows that all of the $P_{f n}$ of our proposed RZW-SR when only utilizing synthesized frames are still very small, with their maximal values as $0.0500,0.0600,0.0550$, and 0.0750 , respectively, and their average values as $0.0092,0.0079,0.0189$, and 0.0114 . For the synthesized frames without attacks, their $P_{f n}$ are $0.0000,0.0000,0.0100$ and 0.0000 . In addition, $P_{f n}$ obtained when fusing the synthesized frames and depth maps are just slightly larger than those when fusing the original 2D frames and depth maps, with their maximal values as $0.0300,0.0400,0.0350$, and 0.0500 and average values as $0.0035,0.0042,0.0046$, and 0.0052. These results demonstrate that the similarity-based retrievals of our proposed RZW-SR when utilizing (fusing) synthesized frames are still effective and that they are just slightly worse than when utilizing (fusing) original 2D frames. The reason for these results is that the features extracted from the original 2D frames and synthesized frames are similar. In addition, the $P_{f n}$ obtained when fusing synthesized frames and depth maps are smaller than those when only utilizing synthesized frames or depth maps, which is consistent with the results listed in Table 3. These results demonstrate that the attention-based fusion also enhances the performances of similarity-based retrieval under DIBR distortions with various attacks.

Finally, we plot detection error trade-off (DET) graphs based on $P_{f n}$ and $P_{f p}$ in Fig. 7 to fully demonstrate the performances of similarity-based retrieval of the proposed RZW-SR. 
Fig. 7 shows that all of the mean $P_{f n}$ are less than 0.2 even when the $P_{f p}$ is set to $10^{-4}$. In addition, all of the DET graphs fusing the 2D frames (synthesized frames) and depth maps are below the graphs when only utilizing 2D frames (synthesized frames) or depth maps, respectively. These results demonstrate that both the accuracy and the reliability of the similarity-based retrieval in RZW-SR are sufficient. In addition, the performance of the similarity-based retrieval is even enhanced by designing the attention-based fusion when the copyrights of 2D frames and depth maps are the same.

TABLE 4. $P_{f n}$ with $P_{f p}=0.01$ under different attacks of our proposed RZW-SR when utilizing depth maps, synthesized frames, and their fusion

\begin{tabular}{|c|c|c|c|c|c|c|c|c|c|}
\hline & \multirow{2}{*}{$\begin{array}{l}\text { Depth } \\
\text { map }\end{array}$} & \multicolumn{4}{|c|}{ Baseline distance $=5 \%$ frame width } & \multicolumn{4}{|c|}{ Baseline distance $=7 \%$ frame width } \\
\hline & & $\begin{array}{l}\text { Synthesized } \\
\text { frame (left) }\end{array}$ & $\begin{array}{l}\text { Two } \\
\text { together }\end{array}$ & $\begin{array}{l}\text { Synthesized } \\
\text { frame (right) }\end{array}$ & $\begin{array}{c}\text { Two } \\
\text { together }\end{array}$ & $\begin{array}{l}\text { Synthesized } \\
\text { frame (left) }\end{array}$ & $\begin{array}{c}\text { Two } \\
\text { together }\end{array}$ & $\begin{array}{l}\text { Synthesized } \\
\text { frame (right) }\end{array}$ & $\begin{array}{c}\text { Two } \\
\text { together }\end{array}$ \\
\hline Without attack & 0.0000 & 0.0000 & 0.0000 & 0.0000 & 0.0000 & 0.0100 & 0.0000 & 0.0000 & 0.0000 \\
\hline GB $9 \times 9$ & 0.0200 & 0.0000 & 0.0000 & 0.0000 & 0.0000 & 0.0100 & 0.0000 & 0.0000 & 0.0000 \\
\hline GB $15 \times 15$ & 0.0200 & 0.0000 & 0.0000 & 0.0000 & 0.0000 & 0.0100 & 0.0000 & 0.0000 & 0.0000 \\
\hline $\mathrm{AF} 9 \times 9$ & 0.0350 & 0.1500 & 0.0000 & 0.0100 & 0.0000 & 0.0200 & 0.0000 & 0.0150 & 0.0000 \\
\hline AF $15 \times 15$ & 0.0450 & 0.0200 & 0.0000 & 0.0100 & 0.0000 & 0.0250 & 0.0000 & 0.0150 & 0.0000 \\
\hline MF $9 \times 9$ & 0.0000 & 0.0100 & 0.0000 & 0.0100 & 0.0000 & 0.0200 & 0.0000 & 0.0150 & 0.0000 \\
\hline MF15×15 & 0.0000 & 0.0200 & 0.0000 & 0.0100 & 0.0000 & 0.0200 & 0.0000 & 0.0150 & 0.0000 \\
\hline $\mathrm{CC}-30$ & 0.0000 & 0.0000 & 0.0000 & 0.0000 & 0.0000 & 0.0100 & 0.0000 & 0.0000 & 0.0000 \\
\hline $\mathrm{CC}+30$ & 0.0150 & 0.0050 & 0.0000 & 0.0000 & 0.0000 & 0.0150 & 0.0000 & 0.0000 & 0.0000 \\
\hline CB -30 & 0.0000 & 0.0100 & 0.0000 & 0.0050 & 0.0000 & 0.0200 & 0.0000 & 0.0050 & 0.0000 \\
\hline $\mathrm{CB}+30$ & 0.0400 & 0.0000 & 0.0000 & 0.0000 & 0.0000 & 0.0100 & 0.0000 & 0.0000 & 0.0000 \\
\hline GT $\gamma=0.6$ & 0.0000 & 0.0000 & 0.0000 & 0.0000 & 0.0000 & 0.0100 & 0.0000 & 0.0000 & 0.0000 \\
\hline GT $\gamma=1.4$ & 0.0000 & 0.0000 & 0.0000 & 0.0000 & 0.0000 & 0.0100 & 0.0000 & 0.0000 & 0.0000 \\
\hline GN 0.005 & 0.0650 & 0.0050 & 0.0000 & 0.0000 & 0.0000 & 0.0150 & 0.0000 & 0.0000 & 0.0000 \\
\hline GN 0.01 & 0.0900 & 0.0100 & 0.0050 & 0.0050 & 0.0050 & 0.0200 & 0.0100 & 0.0050 & 0.0100 \\
\hline LI $32 \times 32$ & 0.0000 & 0.0000 & 0.0000 & 0.0000 & 0.0000 & 0.0100 & 0.0000 & 0.0000 & 0.0000 \\
\hline LI $64 \times 64$ & 0.0400 & 0.0000 & 0.0000 & 0.0000 & 0.0000 & 0.0050 & 0.0000 & 0.0000 & 0.0000 \\
\hline RS $1 / 2$ & 0.0000 & 0.0000 & 0.0000 & 0.0000 & 0.0000 & 0.0100 & 0.0000 & 0.0000 & 0.0000 \\
\hline RS $1 / 5$ & 0.0050 & 0.0100 & 0.0000 & 0.0100 & 0.0000 & 0.0200 & 0.0000 & 0.0150 & 0.0000 \\
\hline CR $5 \%$ & 0.1300 & 0.0050 & 0.0100 & 0.0150 & 0.0150 & 0.0250 & 0.0100 & 0.0150 & 0.0150 \\
\hline CR 10\% & 0.1450 & 0.0200 & 0.0150 & 0.0200 & 0.0150 & 0.0400 & 0.0150 & 0.0300 & 0.0150 \\
\hline RT 45 & 0.1050 & 0.0150 & 0.0100 & 0.0100 & 0.0150 & 0.0300 & 0.0150 & 0.0250 & 0.0200 \\
\hline RT 90 & 0.1450 & 0.0500 & 0.0300 & 0.0600 & 0.0400 & 0.0550 & 0.0350 & 0.0750 & 0.0500 \\
\hline FL Vertical & 0.0000 & 0.0000 & 0.0000 & 0.0000 & 0.0000 & 0.0000 & 0.0000 & 0.0050 & 0.0000 \\
\hline FL Horizontal & 0.0000 & 0.0000 & 0.0000 & 0.0000 & 0.0000 & 0.0100 & 0.0000 & 0.0000 & 0.0000 \\
\hline FR 5\% & 0.1450 & 0.0200 & 0.0100 & 0.0100 & 0.0100 & 0.0300 & 0.0250 & 0.0150 & 0.1500 \\
\hline FD 5\% & 0.0250 & 0.0350 & 0.0150 & 0.0400 & 0.0150 & 0.0500 & 0.0150 & 0.0600 & 0.1500 \\
\hline Average value & 0.0412 & 0.0092 & 0.0035 & 0.0079 & 0.0042 & 0.0189 & 0.0046 & 0.0114 & 0.0052 \\
\hline
\end{tabular}




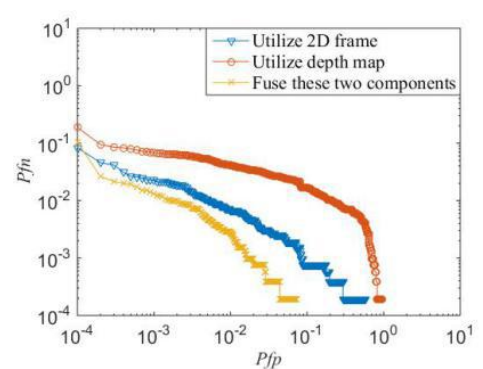

(a)

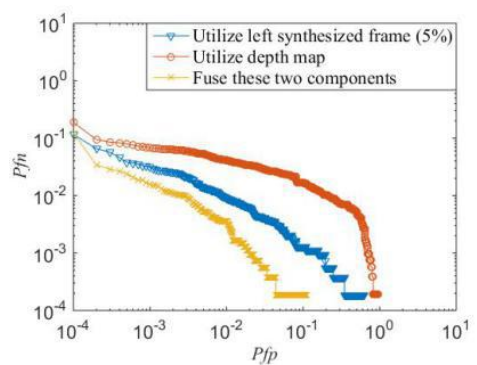

(b)

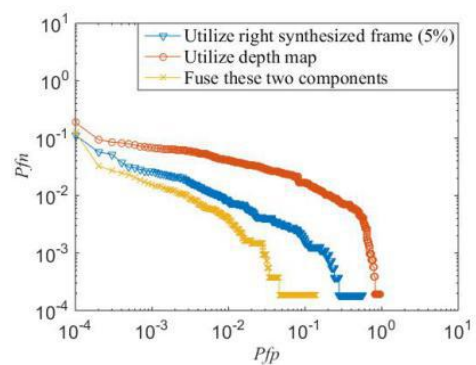

(c)

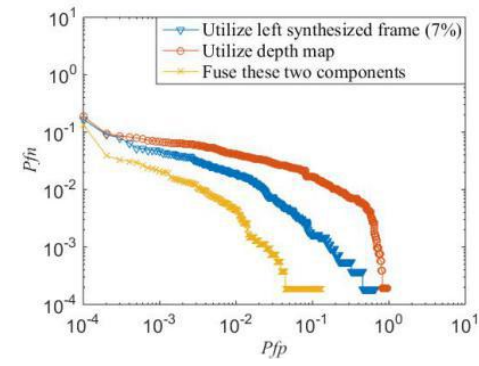

(d)

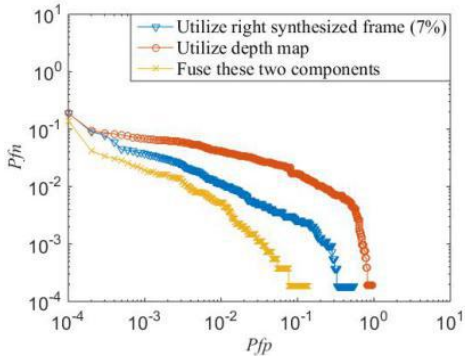

(e)

Fig. 7. DET graphs of the proposed RZW-SR when: (a) utilizing original 2D frames, utilizing depth maps and fusing them; (b) utilizing left synthesized frames (baseline=5\%), utilizing depth maps and fusing them; (c) utilizing right

synthesized frames (baseline $=5 \%$ ), utilizing depth maps and fusing them; (d) utilizing left synthesized frames (baseline $=7 \%$ ), utilizing depth maps and fusing them; and (e) utilizing right synthesized frames (baseline=7\%), utilizing depth maps and fusing them.

Because injective index relationships between the features and ownership shares are established, the ownership share relevant to a particular DIBR 3D video can also be obtained precisely and reliably according to similarity-based retrieval results for the latter copyright identification phase.

\subsection{Evaluation of copyright identification performance}

The performance of copyright identification is mainly considered in terms of accuracy and reliability. For 2D frame and depth map-based watermark schemes, the copyright identification accuracy has been guaranteed because their watermarks are directly embedded into the 3D videos, and the possibility of exacting an accurate watermark from unwatermarked 3D videos is insignificant. For existing zero-watermark schemes, the copyright identification accuracy is not ensured because it is difficult for zero-watermark schemes to obtain the precise ownership share when processing large-scale 3D videos without an efficient indexing mechanism. For our proposed RZW-SR, the accuracy of copyright identification has been ensured in the similarity-based retrieval phase by setting $P_{f p}$ to 0.01 because only the $3 \mathrm{D}$ video that has matching features would be used at the copyright identification phase. In this section, we mainly evaluate the reliability of 
copyright identification by using our proposed RZW-SR. Specifically, we compare our proposed scheme with five distortion-free schemes for 3D videos in terms of watermarking robustness, which are UVW [38], D-NOSE-RW [42], AUEW [29], RZW ${ }_{\text {stereo }}$ [45] and RZW DIBR [30]. Note that lossy watermarking schemes are not included in this section for the fair comparison of watermarking robustness.

A subjective evaluation is first performed for the copyright identification comparison. The watermark images are recovered from both the 2D frames and the depth maps of a typical DIBR 3D video clip 'Ballet' in the open dataset [50] by using our proposed RZW-SR and compared with those detected by using UVW,

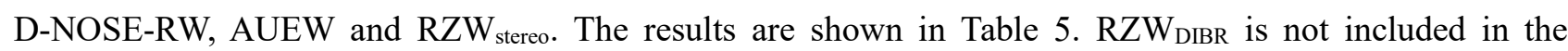
subjective evaluation because the copyright information in RZW DIBR is features of watermark images rather than watermark images themselves, which can hardly be visually presented.

TABLE 5. Subjective evaluation of copyright-identification performances of different schemes

\begin{tabular}{|c|c|c|c|c|c|c|}
\hline & \multirow{2}{*}{$\begin{array}{l}\text { UVW [38] } \\
\text { (Depth map) }\end{array}$} & \multirow{2}{*}{$\begin{array}{c}\text { D-NOSE-RW [42] } \\
\text { (Depth map) }\end{array}$} & \multirow{2}{*}{$\begin{array}{l}\text { AUEW [29] } \\
\text { (Depth map) }\end{array}$} & \multirow{2}{*}{$\begin{array}{c}\mathrm{RZW}_{\text {stere }}[45] \\
\text { (2D frame) }\end{array}$} & \multicolumn{2}{|c|}{ Our proposed RZW-SR } \\
\hline & & & & & 2D frame & Depth map \\
\hline $\begin{array}{l}\text { Embedded } \\
\text { watermark }\end{array}$ & & & 4 & ( & ( & ( \\
\hline $\begin{array}{l}\text { Without } \\
\text { attack }\end{array}$ & 6 & & & (太) & (匀) & (A) \\
\hline GB $9 \times 9$ & & & & (a) & & (A) \\
\hline GB $15 \times 15$ & & & & (소 & $(1)$ & (a) \\
\hline AF $9 \times 9$ & and & & & (a) & & a \\
\hline AF $15 \times 15$ & 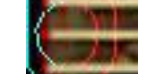 & & & & & (a) \\
\hline MF $9 \times 9$ & & & & (as) & & (A) \\
\hline MF15×15 & & & & & & (a) \\
\hline CC -30 & Gov & & & (a) & (1) & (a) \\
\hline $\mathrm{CC}+30$ & $\operatorname{man}$ & 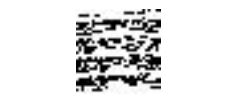 & w & (a) & (1) & (a) \\
\hline CB -30 & ing & 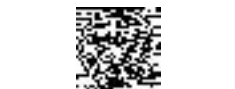 & (a) & $1 \mathrm{a}$ & (A) & (a) \\
\hline $\mathrm{CB}+30$ & 6 & 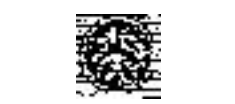 & 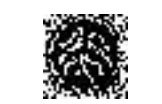 & (a) & 0 & (a) \\
\hline GT $\gamma=0.6$ & and & 解: & (武) & (19) & 6 & (1) \\
\hline
\end{tabular}


TABLE 5. Subjective evaluation of copyright-identification performances of different schemes (continued)

\begin{tabular}{|c|c|c|c|c|c|c|}
\hline & \multirow{2}{*}{$\begin{array}{c}\text { UVW [38] } \\
\text { (Depth map) }\end{array}$} & \multirow{2}{*}{$\begin{array}{c}\text { D-NOSE-RW [42] } \\
\text { (Depth map) }\end{array}$} & \multirow{2}{*}{$\begin{array}{l}\text { AUEW [29] } \\
\text { (Depth map) }\end{array}$} & \multirow{2}{*}{$\begin{array}{l}\mathrm{RZW}_{\text {stereo }}[45] \\
\text { (2D frame) }\end{array}$} & \multicolumn{2}{|c|}{ Our proposed RZW-SR } \\
\hline & & & & & 2D frame & Depth map \\
\hline GT $\gamma=1.4$ & बase & 䀛 & & (a) & (a) & (x) \\
\hline GN 0.005 & 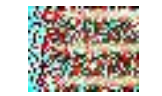 & 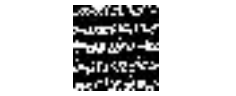 & (6) & (a) & (a) & (a) \\
\hline GN 0.01 & 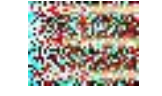 & & 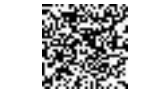 & & a & (a) \\
\hline LI $32 \times 32$ & 62 & (1) & & & (a) & (a) \\
\hline LI $64 \times 64$ & & & & ( & 90 & (a) \\
\hline RS $1 / 2$ & & & & & & (A) \\
\hline RS $1 / 5$ & & & & (a & 19 & (a) \\
\hline CR $5 \%$ & 65e & & & & (a) & (1) \\
\hline CR $10 \%$ & $6 \cos ^{2}$ & (ysis & & & & \\
\hline RT $45^{\circ}$ & & 证 & & 8 & 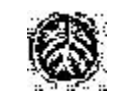 & A \\
\hline RT $90^{\circ}$ & & & & & ats & 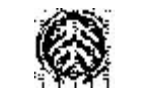 \\
\hline FL Vertical & & & & & & \\
\hline FL Horizonta & $n$ & & & & (1) & (a) \\
\hline FR $5 \%$ & 60 & (永) & & & a & 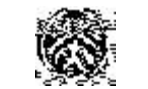 \\
\hline FD 5\% & 6 & ( & & & 柴 & ât \\
\hline
\end{tabular}

As shown in Table 5, watermarks recovered using our proposed RZW-SR are much clearer than those detected using UVW, D-NOSE-RW and AUEW under various attacks. The watermarks in UVW are only viewable but can hardly be extracted. Moreover, the watermarks detected by UVW can hardly be recognized under filtering, noise addition, severe resizing and rotation attacks. The watermarks extracted by D-NOSE-RW can hardly be recognized under filtering, contrast changing, brightness changing, noise addition, resizing, rotation and flipping attacks. The watermarks extracted by AUEW can hardly be recognized under severe filtering, heavy noise addition, rotation and flipping attacks. These results demonstrate that the copyright identification of our proposed RZW-SR is more reliable than those of UVW, 
D-NOSE-RW and AUEW. Although the watermarks recovered by using RZW $\mathrm{W}_{\text {stereo }}$ are also clear under all of the attacks, RZW $\mathrm{W}_{\text {stereo }}$ does not protect the depth maps when the copyrights of the $2 \mathrm{D}$ frames and depth maps are different because its watermarks can only be recovered from the 2D frames. In contrast, our proposed RZW-SR can recover clear watermarks from both the 2D frames and depth maps and can protect these two components independently and reliably, which better satisfies the DRM requirements of DIBR 3D videos.

TABLE 6. Subjective evaluation of copyright-identification performance when utilizing synthesized frames

\begin{tabular}{|c|c|c|c|c|c|c|c|c|c|}
\hline & \multicolumn{2}{|c|}{$\begin{array}{l}\text { Our proposed RZW-SR } \\
\text { (baseline distance }=5 \% \text { ) }\end{array}$} & \multicolumn{2}{|c|}{$\begin{array}{l}\text { Our proposed RZW-SR } \\
\text { (baseline distance }=7 \% \text { ) }\end{array}$} & & \multicolumn{2}{|c|}{$\begin{array}{l}\text { Our proposed RZW-SR } \\
\text { (baseline distance }=5 \% \text { ) }\end{array}$} & \multicolumn{2}{|c|}{$\begin{array}{l}\text { Our proposed RZW-SR } \\
\text { (baseline distance=7\%) }\end{array}$} \\
\hline & $\begin{array}{l}\text { Synthesized } \\
\text { frame (left) }\end{array}$ & $\begin{array}{l}\text { Synthesized } \\
\text { frame } \\
\text { (right) }\end{array}$ & $\begin{array}{l}\text { Synthesized } \\
\text { frame (left) }\end{array}$ & $\begin{array}{l}\text { Synthesized } \\
\text { frame } \\
\text { (right) }\end{array}$ & & $\begin{array}{l}\text { Synthesized } \\
\text { frame (left) }\end{array}$ & $\begin{array}{c}\text { Synthesized } \\
\text { frame } \\
\text { (right) }\end{array}$ & $\begin{array}{l}\text { Synthesized } \\
\text { frame (left) }\end{array}$ & $\begin{array}{l}\text { Synthesized } \\
\text { frame } \\
\text { (right) }\end{array}$ \\
\hline $\begin{array}{l}\text { Embedded } \\
\text { watermark }\end{array}$ & & & & & $\begin{array}{l}\text { Without } \\
\text { attack }\end{array}$ & & & & \\
\hline GB $9 \times 9$ & 隹: & 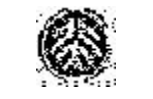 & tots: & 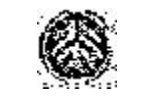 & GB $15 \times 15$ & 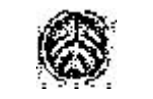 & & & to: \\
\hline AF $9 \times 9$ & 偖: & 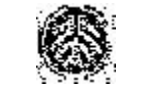 & 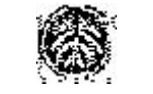 & 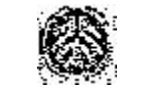 & AF $15 \times 15$ & 触: & 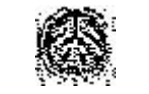 & 解: & 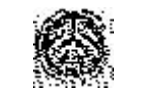 \\
\hline MF $9 \times 9$ & a & 8 & 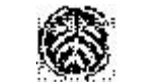 & 8 & MF15×15 & & & & \\
\hline CC -30 & 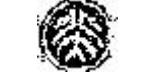 & & & & $\mathrm{CC}+30$ & & & & \\
\hline CB -30 & 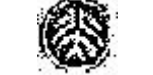 & & 8 & & $\mathrm{CB}+30$ & & & & \\
\hline GT $\gamma=0.6$ & 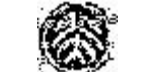 & & 8 & & GT $\gamma=1.4$ & & & & \\
\hline GN 0.005 & 8 & 8 & ( & & GN 0.01 & & & (4) & \\
\hline LI $32 \times 32$ & 4 & & 8 & & LI $64 \times 64$ & $(6 x)$ & & 作 & \\
\hline RS $1 / 2$ & (A) & (6) & 8 & & RS 1/5 & & & & \\
\hline CR 5\% & 40 & (a) & 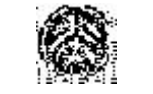 & & CR $10 \%$ & 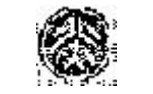 & & 6 & \\
\hline $\mathrm{RT} 45^{\circ}$ & : & 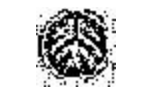 & 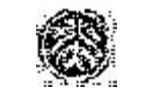 & 4 & RT $90^{\circ}$ & 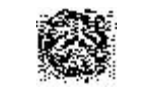 & 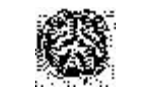 & 触: & \\
\hline FL Vertical & 8 & 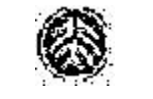 & 4 & & $\begin{array}{l}\text { FL } \\
\text { Horizontal }\end{array}$ & 6 & 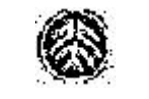 & 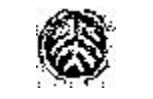 & \\
\hline FR 5\% & $(x+3)$ & 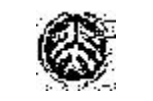 & 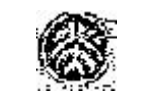 & $(x)$ & FD 5\% & 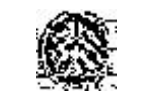 & $\cos _{4}$ & $\cos _{4}$ & 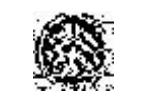 \\
\hline
\end{tabular}

In addition, the watermark images are also recovered from the synthesized frames of 'Ballet' by using our proposed RZW-SR. As shown in Table 6, the watermarks recovered from the synthesized frames using 
our proposed scheme are still clear for copyright identification under all of the attacks, which demonstrates that our proposed RZW-SR can identify the copyrights of 2D frames reliably even when using the DIBR synthesized frames under various attacks.

To further evaluate the copyright-identification reliability of our proposed RZW-SR, objective evaluation is performed by calculating the mean $B E R$ s between the original watermark and watermarks recovered from attacked 3D videos. On the one hand, we calculate the mean BERs of our proposed RZW-SR when utilizing original 2D frames, utilizing depth maps, and fusing these two components together. The

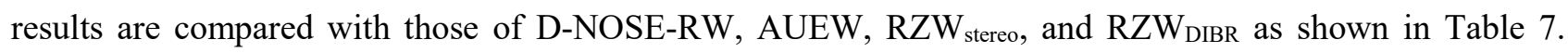
UVW is not included in the subjective evaluation because its watermarks are only viewable but can hardly be extracted for objective evaluation. On the other hand, we calculate the mean BERs of our proposed RZW-SR when utilizing synthesized frames and fusing them with their depth maps to evaluate our watermark robustness against DIBR distortions. The results are shown in Table 8.

Table 7 shows that the mean BERs of our proposed RZW-SR when utilizing original 2D frames, utilizing depth maps and fusing them together are very small. The results demonstrate that the copyright identification of our proposed RZW-SR is reliable under all of these attacks. In addition, the mean BERs when fusing 2D frames and depth maps together are even smaller than those when utilizing either of them alone. The results demonstrate that attention-based fusion enhances the copyright-identification performance. The reason for this phenomenon is the utilization of the complementary feature robustness of 2D frames and depth maps, as analysed in section 4.3.

Moreover, when the copyrights of 2D frames and depth maps are the same, our proposed RZW-SR and RZW DIBR use these two components together. In this situation, the maximal BER of our proposed RZW-SR is 0.0870 , which is still very close to 0 . This value is much smaller than those of D-NOSE-RW, AUEW, and $\mathrm{RZW}_{\text {DIBR, }}$ which are $0.7259,0.5873$ and 0.4772 , and slightly smaller than that of $\mathrm{RZW}_{\text {stereo, }}$ which is 0.1061 . In addition, the average value of the mean BERs of our proposed RZW-SR is merely 0.0287 . This value is much smaller than those of D-NOSE-RW, AUEW, and RZW DIBR, which are 0.4218, 0.1759 and 0.1185 , and slightly smaller than that of $\mathrm{RZW}_{\text {stereo, }}$ which is 0.0312 . These results demonstrate that copyright identification of our proposed RZW-SR is much more reliable than those of D-NOSE-RW, AUEW, and $\mathrm{RZW}_{\text {DIBR }}$ and slightly better than that of $\mathrm{RZW}_{\text {stereo }}$ when the copyrights of $2 \mathrm{D}$ frames and depth maps are the same.

In addition, only our proposed RZW-SR and RZW when the copyrights of 2D frames and depth maps are different. In contrast, D-NOSE-RW and AUEW only 
protect the depth maps, whereas $\mathrm{RZW}_{\text {stereo }}$ only protects the 2D frames. Therefore, RZW-SR and RZW DIBR are more flexible and can better satisfy the requirements for protecting DIBR 3D videos than the other three schemes. Moreover, the maximal mean BERs of 2D frames and depth maps of our proposed RZW-SR are 0.1783 and 0.1700 . These values are much smaller than those of RZW DIBR, which are 0.4981 and 0.4942 . The average $B E R$ values of our proposed RZW-SR are merely 0.0619 and 0.0628 . These values are also much smaller than those of $\mathrm{RZW}$ DIBR, which are 0.1308 and 0.1706 . These results demonstrate that the copyright identification of our proposed RZW-SR is more flexible than those of D-NOSE-RW, AUEW and $\mathrm{RZW}_{\text {stereo }}$ to better satisfy the requirements for protecting DIBR 3D videos and much more reliable than the copyright identification of RZW DIBR.

TABLE 7. Mean $B E R$ s of different schemes

\begin{tabular}{|c|c|c|c|c|c|c|c|c|c|}
\hline & \multirow{2}{*}{$\begin{array}{l}\text { D-NOSE-RW } \\
\text { [42] (Depth } \\
\text { map) }\end{array}$} & \multirow{2}{*}{$\begin{array}{c}\text { AUEW } \\
\text { [29] (Depth } \\
\text { map) }\end{array}$} & \multirow{2}{*}{$\begin{array}{c}\mathrm{RZW}_{\text {stereo }} \\
{[45](2 \mathrm{D}} \\
\text { frame })\end{array}$} & \multicolumn{3}{|c|}{$\mathrm{RZW}_{\text {DIBR }}[30]$} & \multicolumn{3}{|c|}{ Our proposed RZW-SR } \\
\hline & & & & 2D frame & Depth map & $\begin{array}{c}\text { Fuse } \\
\text { together }\end{array}$ & 2D frame & Depth map & $\begin{array}{l}\text { Fuse } \\
\text { together }\end{array}$ \\
\hline GB $9 \times 9$ & 0.5260 & 0.0000 & 0.0103 & 0.0195 & 0.0597 & 0.0144 & 0.0435 & 0.0216 & 0.0144 \\
\hline GB $15 \times 15$ & 0.5260 & 0.0000 & 0.0103 & 0.0206 & 0.0613 & 0.0077 & 0.0435 & 0.0216 & 0.0144 \\
\hline AF $9 \times 9$ & 0.5467 & 0.0007 & 0.0422 & 0.0444 & 0.0922 & 0.0384 & 0.0822 & 0.0360 & 0.0250 \\
\hline AF $15 \times 15$ & 0.5385 & 0.4731 & 0.0738 & 0.0663 & 0.1184 & 0.0578 & 0.1075 & 0.0478 & 0.0331 \\
\hline MF $9 \times 9$ & 0.5381 & 0.0009 & 0.0398 & 0.0336 & 0.0728 & 0.0247 & 0.0605 & 0.0172 & 0.0134 \\
\hline MF15×15 & 0.5483 & 0.4590 & 0.0625 & 0.0573 & 0.1069 & 0.0461 & 0.0818 & 0.0217 & 0.0171 \\
\hline $\mathrm{CC}-30$ & 0.3691 & 0.0005 & 0.0273 & 0.0144 & 0.0408 & 0.0083 & 0.0210 & 0.0217 & 0.0106 \\
\hline $\mathrm{CC}+30$ & 0.3381 & 0.1319 & 0.0019 & 0.0277 & 0.0947 & 0.0233 & 0.0319 & 0.0815 & 0.0229 \\
\hline CB -30 & 0.3909 & 0.0277 & 0.0218 & 0.0264 & 0.0483 & 0.0155 & 0.0698 & 0.0786 & 0.0369 \\
\hline $\mathrm{CB}+30$ & 0.3927 & 0.1343 & 0.0312 & 0.0239 & 0.1022 & 0.0197 & 0.0595 & 0.1068 & 0.0382 \\
\hline GT $\gamma=0.6$ & 0.3915 & 0.0001 & 0.0042 & 0.0492 & 0.0659 & 0.0375 & 0.0463 & 0.0473 & 0.0234 \\
\hline GT $\gamma=1.4$ & 0.3872 & 0.0162 & 0.0291 & 0.0414 & 0.0558 & 0.0178 & 0.0367 & 0.0425 & 0.0197 \\
\hline GN 0.005 & 0.7233 & 0.1615 & 0.0753 & 0.0180 & 0.0422 & 0.0106 & 0.0424 & 0.0922 & 0.0291 \\
\hline GN 0.01 & 0.7259 & 0.4184 & 0.1061 & 0.0206 & 0.0464 & 0.0134 & 0.0632 & 0.1197 & 0.0413 \\
\hline LI $32 \times 32$ & 0.0659 & 0.0009 & 0.0255 & 0.2075 & 0.2797 & 0.1823 & 0.0000 & 0.0115 & 0.0000 \\
\hline LI $64 \times 64$ & 0.1309 & 0.0059 & 0.0171 & 0.2919 & 0.3459 & 0.2648 & 0.0475 & 0.0451 & 0.0231 \\
\hline $\mathrm{RS} 1 / 2$ & 0.5425 & 0.0000 & 0.0007 & 0.0141 & 0.0347 & 0.0067 & 0.0329 & 0.0147 & 0.0101 \\
\hline RS $1 / 5$ & 0.4855 & 0.0004 & 0.0007 & 0.0139 & 0.0342 & 0.0075 & 0.0643 & 0.0220 & 0.0164 \\
\hline CR 5\% & 0.2027 & 0.1086 & 0.0252 & 0.1578 & 0.3308 & 0.1570 & 0.0459 & 0.0650 & 0.0269 \\
\hline CR $10 \%$ & 0.4339 & 0.2139 & 0.0203 & 0.2330 & 0.3625 & 0.2236 & 0.0782 & 0.1099 & 0.0457 \\
\hline RT 45 & 0.4896 & 0.5873 & 0.0355 & 0.4567 & 0.4742 & 0.4394 & 0.1422 & 0.1458 & 0.0719 \\
\hline RT 90 & 0.5224 & 0.5820 & 0.0263 & 0.4898 & 0.4867 & 0.4678 & 0.1783 & 0.1700 & 0.0870 \\
\hline FL Vertical & 0.5561 & 0.5826 & 0.0000 & 0.4981 & 0.4942 & 0.4772 & 0.0112 & 0.0180 & 0.0069 \\
\hline FL Horizontal & 0.5747 & 0.5863 & 0.0602 & 0.4906 & 0.4895 & 0.4720 & 0.0105 & 0.0178 & 0.0066 \\
\hline FR 5\% & 0.0202 & 0.0406 & 0.0324 & 0.0325 & 0.0441 & 0.0180 & 0.0943 & 0.1638 & 0.0598 \\
\hline FD 5\% & 0.0000 & 0.0422 & 0.0315 & 0.0519 & 0.0509 & 0.0298 & 0.1153 & 0.0942 & 0.0518 \\
\hline
\end{tabular}


TABLE 8. Mean $B E R$ s of RZW-SR when utilizing depth maps, utilizing synthesized frames, and fusing them

\begin{tabular}{|c|c|c|c|c|c|c|c|c|c|}
\hline & \multirow{2}{*}{$\begin{array}{l}\text { Depth } \\
\text { map }\end{array}$} & \multicolumn{4}{|c|}{ Baseline distance $=5 \%$ frame width } & \multicolumn{4}{|c|}{ Baseline distance $=7 \%$ frame width } \\
\hline & & $\begin{array}{l}\text { Synthesized } \\
\text { frame (left) }\end{array}$ & $\begin{array}{l}\text { Fuse } \\
\text { together }\end{array}$ & $\begin{array}{l}\text { Synthesized } \\
\text { frame (right) }\end{array}$ & $\begin{array}{l}\text { Fuse } \\
\text { together }\end{array}$ & $\begin{array}{l}\text { Synthesized } \\
\text { frame (left) }\end{array}$ & $\begin{array}{c}\text { Fuse } \\
\text { together }\end{array}$ & $\begin{array}{l}\text { Synthesized } \\
\text { frame (right) }\end{array}$ & $\begin{array}{l}\text { Fuse } \\
\text { together }\end{array}$ \\
\hline Without attack & 0.0000 & 0.0495 & 0.0000 & 0.0492 & 0.0000 & 0.0670 & 0.0000 & 0.0670 & 0.0000 \\
\hline GB $9 \times 9$ & 0.0216 & 0.0692 & 0.0114 & 0.0695 & 0.0115 & 0.0838 & 0.0119 & 0.0844 & 0.0120 \\
\hline GB $15 \times 15$ & 0.0216 & 0.0693 & 0.0114 & 0.0695 & 0.0115 & 0.0838 & 0.0119 & 0.0844 & 0.0120 \\
\hline AF $9 \times 9$ & 0.0360 & 0.0977 & 0.0267 & 0.0975 & 0.0267 & 0.1082 & 0.0272 & 0.1089 & 0.0270 \\
\hline AF $15 \times 15$ & 0.0478 & 0.1182 & 0.0385 & 0.1180 & 0.0389 & 0.1265 & 0.0391 & 0.1264 & 0.0391 \\
\hline MF $9 \times 9$ & 0.0172 & 0.0799 & 0.0086 & 0.0974 & 0.0085 & 0.0928 & 0.0088 & 0.0939 & 0.0087 \\
\hline MF15×15 & 0.0217 & 0.0950 & 0.0136 & 0.0805 & 0.0136 & 0.1053 & 0.0138 & 0.1073 & 0.0135 \\
\hline CC -30 & 0.0217 & 0.0578 & 0.0111 & 0.0581 & 0.0111 & 0.0744 & 0.0117 & 0.0738 & 0.0116 \\
\hline $\mathrm{CC}+30$ & 0.0815 & 0.0645 & 0.0432 & 0.0639 & 0.0430 & 0.0801 & 0.0494 & 0.0786 & 0.0493 \\
\hline CB -30 & 0.0786 & 0.0914 & 0.0563 & 0.0905 & 0.0560 & 0.1044 & 0.0598 & 0.1029 & 0.0589 \\
\hline $\mathrm{CB}+30$ & 0.1068 & 0.0824 & 0.0590 & 0.0820 & 0.0589 & 0.0944 & 0.0646 & 0.0941 & 0.0643 \\
\hline GT $\gamma=0.6$ & 0.0473 & 0.0704 & 0.0305 & 0.0701 & 0.0305 & 0.0846 & 0.0321 & 0.0842 & 0.0326 \\
\hline $\mathrm{GT} \gamma=1.4$ & 0.0425 & 0.0629 & 0.0264 & 0.0641 & 0.0263 & 0.0782 & 0.0279 & 0.0789 & 0.0277 \\
\hline GN 0.005 & 0.0922 & 0.0676 & 0.0477 & 0.0695 & 0.0492 & 0.0832 & 0.0530 & 0.0839 & 0.0543 \\
\hline GN 0.01 & 0.1197 & 0.0834 & 0.0625 & 0.0847 & 0.0644 & 0.0966 & 0.0686 & 0.0976 & 0.0705 \\
\hline LI $32 \times 32$ & 0.0115 & 0.0495 & 0.0017 & 0.0492 & 0.0015 & 0.0670 & 0.0019 & 0.0670 & 0.0018 \\
\hline LI $64 \times 64$ & 0.0451 & 0.0772 & 0.0282 & 0.0755 & 0.0278 & 0.0918 & 0.0294 & 0.0895 & 0.0294 \\
\hline RS $1 / 2$ & 0.0147 & 0.0630 & 0.0053 & 0.0626 & 0.0052 & 0.0788 & 0.0055 & 0.0785 & 0.0055 \\
\hline RS $1 / 5$ & 0.0220 & 0.0841 & 0.0135 & 0.0837 & 0.0132 & 0.0970 & 0.0137 & 0.0971 & 0.0137 \\
\hline CR 5\% & 0.0650 & 0.0769 & 0.0452 & 0.0765 & 0.0457 & 0.0926 & 0.0485 & 0.0916 & 0.0489 \\
\hline CR $10 \%$ & 0.1099 & 0.1017 & 0.0701 & 0.1014 & 0.0700 & 0.1148 & 0.0749 & 0.1144 & 0.0753 \\
\hline RT 45 & 0.1458 & 0.1504 & 0.1056 & 0.1547 & 0.1085 & 0.1568 & 0.1079 & 0.1615 & 0.1104 \\
\hline RT 90 & 0.1700 & 0.1811 & 0.1289 & 0.1860 & 0.1302 & 0.1855 & 0.1306 & 0.1907 & 0.1317 \\
\hline FL Vertical & 0.0180 & 0.0405 & 0.0077 & 0.0584 & 0.0082 & 0.0585 & 0.0081 & 0.0753 & 0.0084 \\
\hline FL Horizontal & 0.0178 & 0.0508 & 0.0078 & 0.0504 & 0.0075 & 0.0679 & 0.0080 & 0.0675 & 0.0078 \\
\hline FR 5\% & 0.1638 & 0.1096 & 0.0896 & 0.1088 & 0.0895 & 0.1205 & 0.0960 & 0.1194 & 0.0961 \\
\hline FD $5 \%$ & 0.0942 & 0.1292 & 0.0787 & 0.1298 & 0.0798 & 0.1382 & 0.0798 & 0.1397 & 0.0811 \\
\hline Average value & 0.0628 & 0.0842 & 0.0381 & 0.0852 & 0.0384 & 0.0975 & 0.0402 & 0.0985 & 0.0404 \\
\hline
\end{tabular}

Table 8 shows that the mean $B E R$ s when utilizing synthesized frames are still very small, with maximal values of $0.1811,0.1860,0.1855$, and 0.1907 and average values of $0.0842,0.0852,0.0975$, and 0.0985 . For the synthesized frames without attacks, the BERs are as small as $0.0495,0.0492,0.0670$ and 0.0670 . In addition, the mean $B E R$ s when fusing the synthesized frames and depth maps are also just slightly larger than when fusing the original 2D frames and depth maps, with maximal values of $0.1289,0.1302,0.1306$, and 0.1317 and average values of $0.0381,0.0384,0.0402$, and 0.0404 . These results demonstrate that copyright 
identification of our proposed RZW-SR when utilizing (fusing) synthesized frames is still remarkable and just slightly worse than utilizing (fusing) original 2D frames. In addition, the BERs when fusing the synthesized frames and depth maps are smaller than those when only utilizing synthesized frames or depth maps, which is consistent with the results listed in Table 6. These results demonstrate that the attention-based fusion also enhances the performances of copyright identification under DIBR distortions with various attacks.

\subsection{Evaluation of computational complexity}

In this section, the computational complexity of our proposed RZW-SR is evaluated and discussed. Our proposed RZW-SR consists of three phases: the copyright registration phase, retrieval phase and copyright identification phase. The first phase is off-line, and the other two phases are on-line. The mean execution CPU times of different functions in different phases for one 3D video clip are tested over all of the 200 video clips. The results are compared with the other two state-of-the-art zero-watermark schemes, which are $\mathrm{RZW}_{\text {stereo }}$ [45] and RZW Intel ${ }^{\circledR}$ Core $^{\mathrm{TM}}$ i7-7700HQ Processor (4 cores at $2.80 \mathrm{GHz}$ ) with $32 \mathrm{~GB}$ of RAM via a MATLAB implementation.

TABLE 9. Mean execution times (s) of different functions of different schemes for one video clip

\begin{tabular}{|c|c|c|c|c|c|c|}
\hline & & & & $\mathrm{RZW}_{\text {stereo }}[45]$ & $\mathrm{RZW}_{\text {DIBR }}[30]$ & $\begin{array}{c}\text { Our proposed } \\
\text { RZW-SR }\end{array}$ \\
\hline \multirow{4}{*}{ Off-line } & \multirow{4}{*}{ Registration phase } & \multirow{2}{*}{ Feature extraction } & 2D frame & $1.57 \times 10^{1}$ & $4.49 \times 10^{-2}$ & $5.36 \times 10^{-1}$ \\
\hline & & & Depth map & NA & $3.75 \times 10^{-2}$ & $5.27 \times 10^{-1}$ \\
\hline & & Generation of master and & $2 \mathrm{D}$ frame & $2.74 \times 10^{-5}$ & $1.65 \times 10^{-4}$ & $3.60 \times 10^{-3}$ \\
\hline & & ownership shares & Depth map & NA & $1.59 \times 10^{-4}$ & $3.59 \times 10^{-3}$ \\
\hline \multirow{8}{*}{ On-line } & & \multirow{3}{*}{ Retrieval phase } & 2D frame & NA & NA & $3.09 \times 10^{-3}$ \\
\hline & & & Depth map & NA & NA & $3.07 \times 10^{-3}$ \\
\hline & & & Fusion function & NA & NA & $7.50 \times 10^{-4}$ \\
\hline & \multirow{5}{*}{ Identification phase } & \multirow{2}{*}{ Feature extraction } & 2D frame & $1.51 \times 10^{1}$ & $4.37 \times 10^{-2}$ & $5.28 \times 10^{-1}$ \\
\hline & & & Depth map & NA & $3.65 \times 10^{-2}$ & $5.21 \times 10^{-1}$ \\
\hline & & \multirow{3}{*}{$\begin{array}{l}\text { Copyright information recovery } \\
\text { and identification }\end{array}$} & 2D frame & $2.71 \times 10^{-5}$ & $2.26 \times 10^{-4}$ & $5.50 \times 10^{-3}$ \\
\hline & & & Depth map & NA & $2.25 \times 10^{-4}$ & $5.40 \times 10^{-3}$ \\
\hline & & & Fusion function & NA & $6.15 \times 10^{-7}$ & $9.03 \times 10^{-6}$ \\
\hline
\end{tabular}

Although the framework of our proposed scheme is slightly more complicated due to the design of the novel retrieval phase, the additional mean CPU execution time of the retrieval phase is only $6.91 \times 10^{-3} \mathrm{~s}(3.09$ $\times 10^{-3} \mathrm{~s}+3.07 \times 10^{-3} \mathrm{~s}+7.50 \times 10^{-4} \mathrm{~s}$ ), which only occupies $0.32 \%$ of the total cost and is insignificant 
compared with the mean CPU execution time of feature extractions, as shown in Table 9. Moreover, the use of the retrieval phase is necessary because it ensures the copyright identification accuracy when processing large-scale videos, which cannot be achieved by the other two zero-watermark schemes.

In addition, the most time-consuming functions of zero-watermark schemes are the feature extractions, which occupy almost $99 \%$ of the total costs $\left(99.99 \%\right.$ for $\mathrm{RZW}_{\text {stereo, }} 99.51 \%$ for $\mathrm{RZW}$ DIBR and $98.83 \%$ for RZW-SR). Therefore, the mean CPU execution time is mainly determined by the computational complexity of feature extraction. Although the CPU execution times of feature extraction in RZW $\mathrm{W}_{\text {DIBR }}[30]$ are shorter than those of RZW stereo $_{\text {[45] }}$ and our proposed RZW-SR, the robustness of the simple 2D-DCT-based feature in RZW DIBR is extremely poor against geometrical attacks such as cropping, rotation, and flipping, and the copyright identification using RZW DIBR will fail under these attacks, as shown in Table 7. Therefore, it is necessary to design a more robust and complicated feature to ensure higher copyright identification reliability. Both the features in $\mathrm{RZW}_{\text {stereo }}$ and our proposed RZW-SR guarantee the copyright identification reliability under signal, geometrical and temporal attacks. Compared with $\mathrm{RZW}_{\text {stereo, }}$, the CPU execution times for extracting our designed feature are much shorter (less than $1 / 10$ of those of $\mathrm{RZW}_{\text {stereo}}$ ), and our designed feature is much more discriminative.

In summary, our proposed RZW-SR ensures sufficient copyright identification accuracy and reliability with acceptable CPU execution time and thus achieves better trade-off between the DRM performance and computational complexity than the two state-of-the-art zero-watermark schemes

\subsection{Qualitative comparisons with current state-of-the-art schemes}

In this section, qualitative comparisons with other state-of-the-art watermark schemes are conducted in terms of 5 aspects: 1) reliability of the copyright identification, 2) accuracy of the copyright identification, 3) distortion of DIBR 3D video, 4) involvement of similarity-based retrieval, and 5) independence of the copyrights of the 2D frames and depth maps. The comparison results are shown in Table 10.

TABLE 10. Qualitative comparisons with other schemes

\begin{tabular}{|c|c|c|c|c|c|}
\hline & \multirow{2}{*}{$\begin{array}{c}\text { 2D frame-based } \\
\text { watermark }\end{array}$} & \multirow{2}{*}{$\begin{array}{c}\text { Depth map-based } \\
\text { watermark }\end{array}$} & \multicolumn{2}{|c|}{ Zero-watermark } & \multirow{2}{*}{$\begin{array}{c}\text { Our proposed } \\
\text { RZW-SR }\end{array}$} \\
\hline & & & $\mathrm{RZW}_{\text {Stereo }}$ & RZW & \\
\hline Reliability & Sufficient & Insufficient & Sufficient & Sufficient & Sufficient \\
\hline Accuracy & Sufficient & Sufficient & Insufficient & Insufficient & Sufficient \\
\hline Distortion of 3D video & Not free & Free & Free & Free & Free \\
\hline Similarity-based retrieval & No & No & No & No & Yes \\
\hline Independence of copyrights & No & No & No & Yes & Yes \\
\hline
\end{tabular}


As shown in Table 10, our proposed RZW-SR outperforms other state-of-the-art schemes in terms of all 5 of these aspects. In contrast to $2 \mathrm{D}$ frame-based watermark schemes $[1-3,6-7,9,13,20,23,26,31,34,36$, 47], RZW-SR does not directly embed watermark information into 2D frames, which avoids the introduction of distortion into the $3 \mathrm{D}$ videos. Compared with the depth map-based watermark schemes $[8,28-29,33$, 37-38, 42], RZW-SR is more robust against various video attacks and thus can provide more reliable copyright identification. Compared with the other two zero-watermark schemes, which are RZW $\mathrm{W}_{\text {Stereo }}$ [45] and RZW ${ }_{\text {DIBR }}$ [30], RZW-SR designs an effective similarity-based retrieval mechanism, and their relevant ownership can be obtained precisely according to the retrieval results. Therefore, the accuracy of copyright identification in RZW-SR is ensured when processing large-scale videos, which cannot be achieved by other zero-watermark schemes. Last but not least, compared with 2D frame-based watermark schemes, depth map-based watermark schemes and RZW $\mathrm{W}_{\text {Stereo, }}$ only RZW $\mathrm{W}_{\text {DIBR }}$ and our proposed RZW-SR have designed flexible mechanisms for copyright identification to protect the 2D frames and depth maps separately and independently when the copyrights of 2D frames and depth maps are different.

\section{Conclusions}

In this paper, a novel RZW-SR is proposed by fusing the similarity-based retrieval and zero-watermark technique. It is the first scheme that fuses these two techniques to protect DIBR 3D videos. Our analytical and experimental results have demonstrated that our proposed RZW-SR possesses the following merits. First, RZW-SR does not directly embed the copyright information into the $3 \mathrm{D}$ videos. Therefore, RZW-SR can avoid content distortion, which outperforms 2D frame-based watermark schemes. Second, RZW-SR achieves significant robustness against signal, geometrical, and temporal attacks and DIBR distortions by utilizing temporal information and designing invariant video features, which outperforms depth map-based watermark schemes. Third, RZW-SR can retrieve and protect 2D frames and depth maps of 3D videos independently or simultaneously by designing flexible mechanisms based on attention-based fusion. It better satisfies different DRM requirements of 3D videos compared to $2 \mathrm{D}$ frame-based schemes, depth map-based schemes and

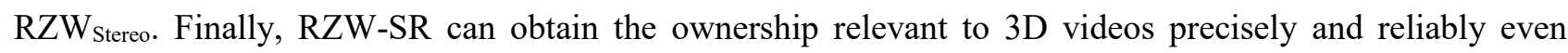
when processing large-scale DIBR 3D videos by designing an effective similarity-based retrieval mechanism, which cannot be achieved by the existing zero-watermark schemes for DIBR 3D videos. In the future, we will further explore and make use of the relationships between video frames, such as the frame variations, to further enhance the feature distinguishability. In addition, exploration of the possibility of applying our proposed scheme to protect medical volume data, such as 3D computed tomography (CT) imaging, magnetic 
resonance imaging (MRI) and optical coherence tomography (OCT) imaging, is also included in our future works.

\section{Acknowledgements}

This research is supported by the National Nature Science Foundations of China [61602527, 61702558, 61772555, 61772553] and the Hunan Provincial Natural Science Foundations of China [2020JJ4746, 2017JJ3416, 2017JJ3411, 2018JJ2548]. The authors are grateful for the support from the postdoctoral research station of Central South University and the valuable comments on this paper from Prof. Shuo Li, Prof. Qi Tian and Dr. Guibo Luo.

\section{References}

[1]. A. Al-Haj, M. E. Farfoura, A. Mohammad, Transform-based watermarking of 3D depth-image-based-rendering images, Measurement 95 (2017) 405-417.

[2]. M. Asikuzzaman, M. J. Alam, A. J. Lambert, and M. R. Pickering, A blind watermarking scheme for depth-image-based rendered 3D video using the dual-tree complex wavelet transform, 2014 IEEE International Conference on Image Processing (ICIP). IEEE, (2014) 5497-5501.

[3]. M. Asikuzzaman, M. J. Alam, A. J. Lambert, and M. R. Pickering, Robust DT CWT-based DIBR 3D video watermarking using chrominance embedding, IEEE T. Multimedia 18 (2016) 1733-1748.

[4]. M. Asikuzzaman, M. R. Pickering, An overview of digital video watermarking, IEEE T. Circ. Syst. Vid. 28 (2018) 2131-2153.

[5]. C. Cheng, C. T. Li, P. S. Huang, T. K. Lin, Y. M. Tsai, and L. G. Chen, A block-based 2D-to-3D conversion system with bilateral filter, 2009 Digest of Technical Papers International Conference on Consumer Electronics. IEEE (2009) $1-2$

[6]. Cui, S. Wang, X. Niu, A novel watermarking for DIBR 3D images with geometric rectification based on feature points, Multimed. Tools Appl. 76 (2017) 649-677.

[7]. Cui, X. M. Niu, A robust DIBR 3D image watermarking algorithm based on histogram shape, Measurement 92 (2016) $130-143$.

[8]. K. L. Chung, W. J. Yang, W. N. Yang, Reversible data hiding for depth maps using the depth no-synthesis-error model, Inform. Sciences 269 (2014) 159-175.

[9]. L. Chen, J. Zhao, Robust contourlet-based blind watermarking for depth-image-based rendering 3D images, Signal 
Process.-Image 54 (2017) 56-65.

[10]. S. C. Chuang, C. H. Huang, and J. L. Wu, Unseen visible watermarking, In: Proc. Int. Conf. Image Processing, IEEE (ICIP), USA, 2007, pp. 261-264.

[11]. M. M. Esmaeili, M. Fatourechi, R. K. Ward, A robust and fast video copy detection system using content-based fingerprinting, IEEE T. Inf. Foren. Sec. 6 (2011) 213-226.

[12]. C. Fehn, K. Schuur, I. Feldmann, P Kauff, and A. Smolic, Distribution of ATTEST test sequences for EE4 in MPEG 3DAV, ISO/IEC JTC1/SC29/WG11 MPEG02/M9219 (2002),2002.

[13]. S. Fan, M. Yu, G. Jiang, F. Shao, and Z. Peng, A digital watermarking algorithm based on region of interest for 3D image, In: Proc. IEEE Int. Conf. Computational Intelligence and Security (CIS), China, 2012, pp. 549-552.

[14]. C. Gong, D. Tao, S. J. Maybank, W. Liu, G. Kang, J. Yang, Multi-modal curriculum learning for semi-supervised image classification, IEEE T. Image Process 25 (2016) 3249-3260.

[15]. C. Gong, D. Tao, X. Chang, J. Yang, Ensemble teaching for hybrid label propagation, IEEE T. Cybernetics 49 (2017) 388-402.

[16]. C. Gong, D. Tao, W. Liu, L. Liu, J. Yang, Label propagation via teaching-to-learn and learning-to-teach, IEEE T. Neur Netlear 28 (2016) 1452-1465.

[17]. C. H. Huang, S. C. Chuang, Y. L. Huang, and J. L. Wu, Unseen visible watermarking: A novel methodology for auxiliary information delivery via visual contents, IEEE T. Inf. Foren. Sec. 4 (2009) 193-206.

[18]. X. S. Hua, H. J. Zhang, An Attention-based fusion Scheme for Multimedia Information Retrieval, In: Proc. Advances in Multimedia Information Processing (PCM), Japan, 2004, pp.1001-1010.

[19]. S. Lee, C. D. Yoo, Robust video fingerprinting for content-based video identification, IEEE T. Circ Syst Vid 18 (2008) 983-988.

[20]. H. D. Kim, J. W. Lee, T. W. Oh, and H. K. Lee, Robust DT-CWT watermarking for DIBR 3D images, IEEE T. Broadcast 58 (2012) 533-543.

[21]. H. J. Kim, V. Sachnev, Y. Q. Shi, J. Nam, and H. G. Choo, A novel difference expansion transform for reversible data embedding, IEEE T. Inf. Foren. Sec. 3 (2008) 456-465.

[22]. P. Kauff, N. Atzpadin, C. Fehn, M. Müller, O. Schreer, A. Smolic, and R. Tanger, Depth map creation and image-based rendering for advanced 3DTV services providing interoperability and scalability, Signal Process.-Image 22 (2007) 217-234.

[23]. W. H. Kim, J. U. Hou, H. U. Jang, and H. K. Lee, Robust template-based watermarking for DIBR 3D images, Appl. Sci. 8 (2018) 911.

[24]. C. C. Lin, W. L. Tai, C. C. Chang, Multilevel reversible data hiding based on histogram modification of difference 
images, Pattern Recogn. 41 (2008) 3582-3591.

[25].L. Li, X. Yuan, Z. Lu, and J. S. Pan, Rotation invariant watermark embedding based on scale-adapted characteristic regions, Inform. Sciences 180 (2010) 2875-2888.

[26]. M. J. Lee, J. W. Lee, and H. K. Lee, Perceptual watermarking for 3D stereoscopic video using depth information, 2011 Seventh International Conference on Intelligent Information Hiding and Multimedia Signal Processing. IEEE (2011) 81-84.

[27]. Q. Liu, Z. Zha, Y. Yang. Gradient-domain-based enhancement of multi-view depth video, Inform. Sciences 281 (2014) 750-761.

[28]. W. Liu, R. Ni, Y. Zhao, Reversible 3D Image Data Hiding with Quality Enhancement, International Workshop on Digital Watermarking. Springer, Cham, (2016) 446-455.

[29]. X. Liu, F. Li, J. Du, Y. Guan, Y. Zhu, and B. Zou, A robust and synthesized-unseen watermarking for the DRM of DIBR-based 3D video, Neurocomputing 222 (2017) 155-169.

[30]. X. Liu, R. Zhao, F. Li, S. Liao, Y. Ding, and B. Zou, Novel robust zero-watermarking scheme for digital rights management of 3D videos, Signal Process.-Image 54 (2017) 140-151.

[31]. Y. H. Lin, J. L. Wu, A digital blind watermarking for depth-image-based rendering 3D images, IEEE T. Broadcast 57 (2011) 602-611.

[32]. Y. H. Lin, J. L. Wu, A depth information based fast mode decision algorithm for color plus depth-map 3D videos, IEEE T. Broadcast 57 (2011) 542-550.

[33]. Y. H. Lin, J. L. Wu, Unseen visible watermarking for color plus depth map 3d images, In: Proc. IEEE Int. Conf. Acoustics, Speech and Signal Processing (ICASSP), Japan, 2012, pp. 1801-1804.

[34]. Y. S. Lee, Y. H. Seo, D. W. Kim, Digital blind watermarking based on depth variation prediction map and DWT for DIBR free-viewpoint image, Signal Process.-Image 70 (2019) 104-113.

[35]. M. Naor, A. Shamir, Visual cryptography, In: Proc. Advances in Cryptology -EUROCRYPT'94, Lecture Notes in Computer Science, Italy, 1994, pp. 1-12.

[36]. S. H. Nam, W. H. Kim, S. M. Mun, J. U. Hou, S. Choi, and H. K. Lee, A SIFT features based blind watermarking for DIBR 3D images, Multimed. Tools Appl. 77 (2018) 7811-7850.

[37]. S. C. Pei, Y. Y. Wang, A new 3D unseen visible watermarking and its applications to multimedia, In: Proc. IEEE Int. Conf. Consumer Electronics (GCCE), Japan, 2014, pp.140-143.

[38]. S. C. Pei, Y. Y. Wang, Auxiliary metadata delivery in view synthesis using depth no synthesis error model, IEEE T. Multimedia 17 (2015) 128-133.

[39]. S. Pereira, T. Pun, Robust template matching for affine resistant image watermarks, IEEE T. Image Process 9 
(2000) 1123-1129.

[40]. A. Redert, M. O. de Beeck, C. Fehn, W. Ijsselsteijn, M. Pollefeys, L. Van Gool, E. Ofek, I. Sexton, and P. Surman, Advanced three-dimensional television system techniques, In: Proc. Int. Symp. 3D Data Processing Visualization and Transmission, Padova, Italy, 2002, pp. 313-319.

[41]. R. Rzeszutek, R. Phan, and D. Androutsos, Semi-automatic synthetic depth map generation for video using random walks, In: Proc. Int. Conf. Multimedia and Expo (ICME), Spain, 2011, pp. 1-6.

[42]. X. Shi, B. Ou, Z. Qin, Tailoring reversible data hiding for 3D synthetic images, Signal Process.-Image 64 (2018) 46-58.

[43]. D.M. Thodi, and J.J. Rodriguez, Prediction-error based reversible watermarking, In: Proc. Int. Conf. on Image Processing (ICIP), Singapore, 2004, pp. 1549-1552.

[44]. R. G. Van Schyndel, A. Z. Tirkel, and C. F. Osborne, A digital watermark, In: Proc. Int. Conf. on Image Processing (ICIP), USA, 1994, pp. 86-90.

[45]. C. Wang, X. Wang, Z. Xia, and C. Zhang, Ternary radial harmonic Fourier moments based robust stereo image zero-watermarking algorithm, Inform. Sciences 470 (2019) 109-120.

[46]. J. Wang, S. Wang, Z. Wang, Asymmetrically compressed stereoscopic 3D videos: Quality assessment and rate-distortion performance evaluation, IEEE T. Image Process 26 (2017) 1330-1343.

[47]. S. Wang, C. Cui, X. Niu, A novel DIBR 3D Image watermarking algorithm resist to geometrical attacks, Chinese J. Electron 26 (2017) 1184-1193.

[48]. Y. Yang, X. Wang, Q. Liu, M. Xu, and L. Yu, A bundled-optimization model of multiview dense depth map synthesis for dynamic scene reconstruction, Inform. Sciences 320 (2015) 306-319.

[49]. J. Yu, D. Tao, M. Wang, Y. Rui, Learning to rank using user clicks and visual features for image retrieval, IEEE T. Cybernetics 45 (2014) 767-779.

[50]. C. L. Zitnick, S. B. Kang, M. Uyttendaele, S. Winder, and R. Szeliski, High-quality video view interpolation using a layered representation, ACM. T. Graphic (TOG). 23 (2004) 600-608. 

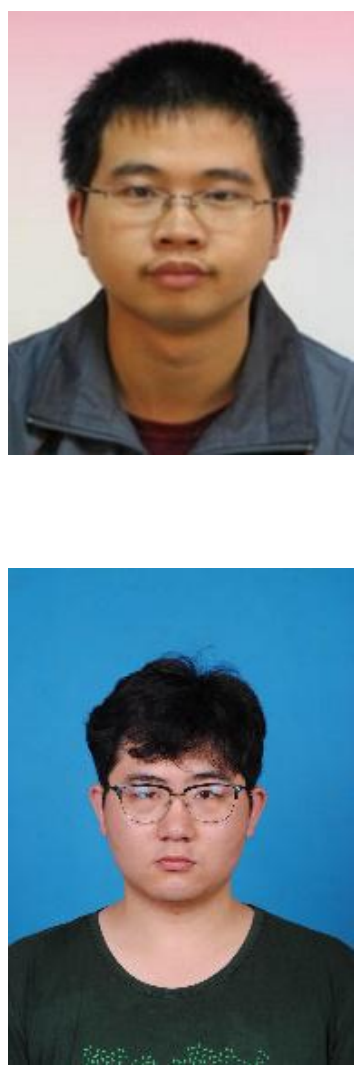

Yifan Wang was born in Hunan Province, in 1994. He received the B.S. degree in Information Management and Information System from Beijing Technology and Business University in 2016. He is currently a postgraduate in Central South University. His research interests include information security and multimedia technology.

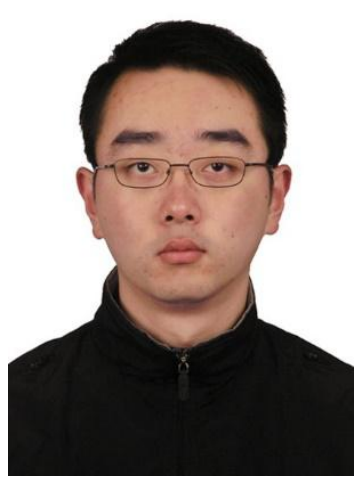

Ziqiang Sun received the B.S. degree from Lanzhou University in 2009, and the Ph.D. degree from Peking University, 2016. He is currently working as an engineer at Nanjing Research Institute of Electronics Technology, China. His research interests include sensor management, target tracking, artificial intelligence, and multimedia technology.

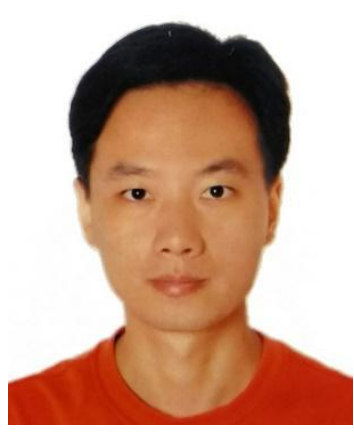

Lei Wang was born in Henan Province, in 1978. He received the B.S. degree from College of Chemistry and Chemical Engineering, Hunan University in 2000 and Ph.D. degree from School of Computer and Communication, Hunan University in 2007. He is now a lecturer in School of Computer Science and Engineering, Central South University, Changsha, China. His research interests include computer vision, multimedia technology and medical image analysis.

Rongchang Zhao received his Ph.D degree in School of Information Science and Engineering from Lanzhou University in 2011. He is now an associate professor in School of Computer Science and Engineering, Central South University, Changsha, 
China. His research interests include multimedia technology, medical image analysis, pattern recognition and artifical intelligence.

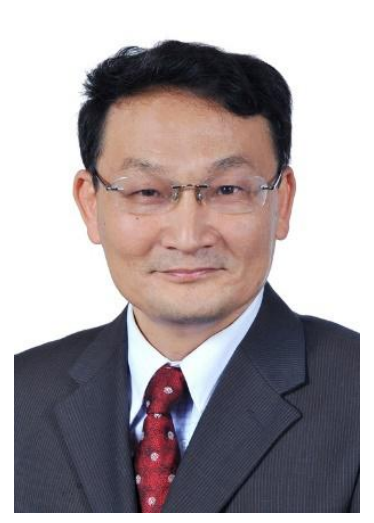

Yuesheng Zhu received his B.Eng. degree in Radio Engineering, M.Eng. degree in Circuits and Systems and Ph.D. degree in Electronics Engineering in 1982, 1989 and 1996, respectively. He is currently working as a professor at the Lab of Communication and Information Security, Shenzhen Graduate School, Peking University. He is a senior member of IEEE, fellow of China Institute of Electronics, and senior member of China Institute of Communications. His interests include digital signal processing, multimedia technology, and information security.

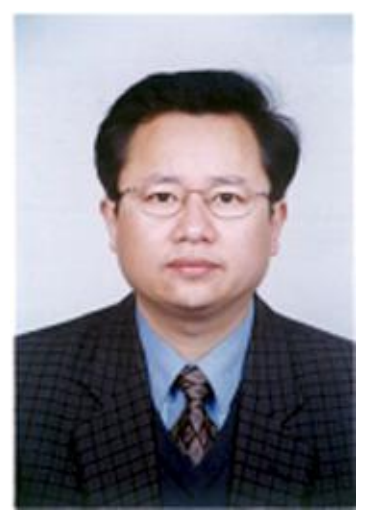

Beiji Zou received the B.S. degree in Computer Science from Zhejiang University, China, in 1982, received the M.S. degree from Tsinghua University specializing CAD and computer graphics in 1984, and obtained the Ph.D. degree from Hunan University in the field of control theory and control engineering in 2001 . He is now a professor in School of Computer Science and Engineering, Central South University, Changsha, China. His research interests include computer graphics, computer vision, multimedia processing and medical image processing.

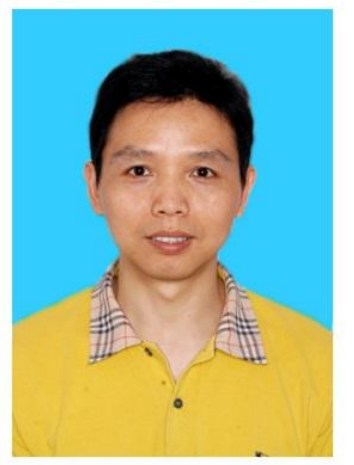

Yuqian Zhao received the B.S. degree in 1997, M.S. degree in 2002, and Ph.D. degree in 2006 from Central South University, Changsha, China, and was awarded New Century Excellent Talents in University of Education Ministry in China in 2013. He is currently a Professor in the School of Automation, Central South University, China. His research interests include machine learning, computer vision, pattern recognition, image processing and analysis, computer-aided diagnosis and surgery, medical AI, video text detection, and image forensics.

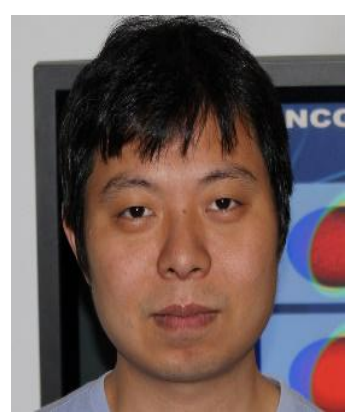

Hui Fang received the B.S. degree from University of Science and Technology, Beijing, China, in 2000 and the Ph.D. degree from the University of Bradford, U.K., in 2006. He is currently with the computer science department, Loughborough University. His research interests include computer vision, image/video processing, 
pattern recognition, machine learning, deep neural networks, data mining, scientific visualization and visual analytics. 